Pure and Applied Mathematics Quarterly

Volume 2, Number 4

(Special Issue: In honor of

Robert MacPherson, Part 2 of 3)

963-1013, 2006

\title{
Alcove Walks, Hecke Algebras, Spherical Functions, Crystals and Column Strict Tableaux
}

\author{
Arun Ram \\ Dedicated to R. MacPherson on the occasion of his 60th birthday
}

\begin{abstract}
The main part of this paper is a survey on crystal bases, the Littelmann path model, the affine Weyl group and the affine Hecke algebra. The new results of this paper are in sections 3 and 4, where a tight connection affine Hecke algebra and path models is established. The key new idea is due to $\mathrm{C}$. Schwer [Sc], who noticed that the periodic Hecke module defined by G. Lusztig encodes the combinatorics of the positively folded galleries used by Gaussent and Littelmann [GL]. H. Pittie and A. Ram [PR] discovered the $q^{-1}=0$ version of this connection when they established a relation between the Littelmann path model and the K-theory of flag varieties. The generalised path model studied in this paper provides a $q$-version of the theory of crystals which is a combinatorics for the spherical functions for a $p$-adic group. This combinatorics specialises, at $q^{-1}=0$, to the path model combinatorics for working with Weyl characters.
\end{abstract}

\section{INTRODUCTION}

Together, Sections 2 and 5 of this paper form a self contained treatment of the theory of crystals and the path model. It is my hope that this will be useful to the many people who, over the years, have told me that they wished they understood crystals but have found the existing literature too daunting. One goal of the presentation here is to clarify the relationship between the general path model and the crystal operators of Lascoux and Schützenberger used in the type A case [LS]. More specifically, Section 2 is a basic pictorial exposition of Weyl groups and affine Weyl groups and Section 5 is an exposition of the theory of (a) symmetric functions, (b) crystals and (c) the path model which is designed for

Received December 13, 2005. 
readers whose only background is the material in Section 2. These two sections can be read independently of Sections 3 and 4 .

Sections 3 and 4 give an exposition of the affine Hecke algebra and recent results regarding the combinatorics of spherical functions on p-adic groups (HallLittlewood polynomials) using only the material in Section 2 as background. The $q$-analogue of the theory of crystals developed in Section 4 specializes to the path model version [Li1-3] of the "classical" crystal theory at $q^{-1}=0$. This specialization property is "to be expected" since Macdonald's formula for the spherical function specializes to the Weyl character formula at $q^{-1}=0$. It is my hope that the presentation of the results will illustrate the close connection between the affine Hecke algebra, the path model, and the theory of crystals.

The motivation for this paper is the following. The classical path model is a combinatorial tool for working with crystal bases of integrable representations of symmetrizable Kac-Moody algebras, a generalization of column strict Young tableaux. The same combinatorics can be used to study the equivariant K-theory of the flag variety $[\mathrm{PR}]$ and this point provides a translation between the path model and the structure of the nil-affine Hecke algebra. Thus, conceptually,

$$
\begin{aligned}
\text { crystal bases } & =\text { the path model } \\
& =\text { the nil affine Hecke algebra } \\
& =\text { the } T \text {-equivariant } K \text {-theory of } G / B .
\end{aligned}
$$

The paper of Gaussent-Littelmann [GL] (see also the work of Kapovich-Millson in $[\mathrm{KM}]$ ) indicated that there is a refined model which removes the 'nil' in this chain of equalities and models the geometry of Kac-Moody groups over a local field. The result is the conceptual chain of equalities

positively folded alcove walks $=$ geodesics in the affine building

$$
\begin{aligned}
& =\text { the affine Hecke algebra } \\
& =\text { MV cycles in the loop Grassmanian. }
\end{aligned}
$$

The connection to the affine Hecke algebra and the approach to spherical functions for a $p$-adic group in $[\mathrm{NR}]$ was made concrete by C. Schwer [Sc] who told me that "the periodic Hecke module encodes the positively folded galleries".

The main results of this paper are obtained by viewing the affine Hecke algebra in terms of a new construct, the alcove walk algebra. This combinatorics provides

(a) a change of basis formula in the affine Hecke algebra which is an analogue of the change of basis formula for the nil affine Hecke algebra given in [GR],

(b) a derivation of the combinatorial formula for the Hall-Littlewood polynomials in terms of monomials given by Schwer $[\mathrm{Sc}]$, 
(c) a derivation of the product formula for Hall-Littlewood polynomials given by Kapovich-Millson [KM] and Schwer [Sc],

(d) a description of the restriction rule for Hall-Littlewood polynomials

in the same way that the theory of crystal bases gives expansions for Weyl characters (Schur functions) in terms of monomials and produces general "LittlewoodRichardson rules" for Weyl characters. The result in (a) is a generalization of a result given in Schwer in the same spirit as the $q^{-1}=0$ result given in the paper of Lenart-Postnikov [LP]. Thus (a) generalizes both the result in [Sc] and the result in [LP]. Results (b) and (c) are all either explicit or implicit in [GL], [KM] and $[\mathrm{Sc}]$. The result in $(\mathrm{d})$ has not appeared in the literature before but it is an easy consequence of the combination of the techniques of $[\mathrm{Sc}]$ and the "crystal structure" on positively folded galleries implicit in [GL]. The q-crystal structure gives easy proofs of the results in (c) and (d).

Unfortunately, the limitations of time and space have not allowed me to provide an exposition of the connection between the alcove walk combinatorics and the geometry of the loop group (which is implicit in the affine Hecke algebra and explicit in $[\mathrm{Mac}],[\mathrm{BD}],[\mathrm{GL}],[\mathrm{KM}]$ and $[\mathrm{Sc}])$. Hopefully this will be done soon in a future paper.

Acknowledgments. I thank P. Littelmann for useful conversations and C. Schwer for teaching me the right way to think about positively folded galleries. I thank the National Science Foundation (grants DMS-0353038 and DMS-0097977), the Vilas Foundation and the Max-Planck-Institut für Mathematik for support of this work. I thank my hosts in Rome, C. DeConcini, C. Procesi and E. Strickland, for their hospitality during a sabbatical when the writing of this paper was completed. It is a pleasure to dedicate this paper to R. MacPherson. He is an inspiration and a role model.

\section{The AfFine Weyl Group}

This section is a summary of the main facts and notations that are needed for working with the affine Weyl group $\tilde{W}$. The main point is that the elements of the affine Weyl group can be identified with alcoves via the bijection in (2.11).

Let $\mathfrak{h}_{\mathbb{R}}^{*}$ be a finite dimensional vector space over $\mathbb{R}$. A reflection is a diagonalizable element of $G L\left(\mathfrak{h}_{\mathbb{R}}^{*}\right)$ which has exactly one eigenvalue not equal to 1 . A lattice is a free $\mathbb{Z}$-module. A Weyl group is a finite subgroup $W$ of $G L\left(\mathfrak{h}_{\mathbb{R}}^{*}\right)$ which is

generated by reflections and acts on a lattice $L$ in $\mathfrak{h}_{\mathbb{R}}^{*}$ 
such that $\mathfrak{h}_{\mathbb{R}}^{*}=L \otimes_{\mathbb{Z}} \mathbb{R}$. Let $R^{+}$be an index set for the reflections in $W$ so that, for $\alpha \in R^{+}$,

$$
s_{\alpha} \text { is the reflection in the hyperplane } H_{\alpha}=\left(\mathfrak{h}_{\mathbb{R}}^{*}\right)^{s_{\alpha}},
$$

the fixed point space of the transformation $s_{\alpha}$. The chambers are the connected components of the complement

$$
\mathfrak{h}_{\mathbb{R}}^{*} \backslash\left(\bigcup_{\alpha \in R^{+}} H_{\alpha}\right)
$$

of these hyperplanes in $\mathfrak{h}_{\mathbb{R}}^{*}$. These are fundamental regions for the action of $W$.

Let $\langle$,$\rangle be a nondegenerate W$-invariant bilinear form on $\mathfrak{h}_{\mathbb{R}}^{*}$. Fix a chamber $C$ and choose vectors $\alpha^{\vee} \in \mathfrak{h}_{\mathbb{R}}^{*}$ such that

$$
C=\left\{x \in \mathfrak{h}_{\mathbb{R}}^{*} \mid\left\langle x, \alpha^{\vee}\right\rangle>0\right\} \quad \text { and } \quad P \supseteq L \supseteq Q
$$

where, with $\alpha=\frac{2 \alpha^{\vee}}{\left\langle\alpha^{\vee}, \alpha^{\vee}\right\rangle}$,

$$
P=\left\{\lambda \in \mathfrak{h}_{\mathbb{R}}^{*} \mid\left\langle\lambda, \alpha^{\vee}\right\rangle \in \mathbb{Z}\right\} \quad \text { and } \quad Q=\sum_{\alpha \in R^{+}} \mathbb{Z} \alpha
$$

Pictorially,

$\left\langle\lambda, \alpha^{\vee}\right\rangle$ is the distance from $\lambda$ to the hyperplane $H_{\alpha}$.

The alcoves are the connected components of the complement

$\mathfrak{h}_{\mathbb{R}}^{*} \backslash\left(\bigcup_{\substack{\alpha \in R^{+} \\ j \in \mathbb{Z}}} H_{\alpha, j}\right) \quad$ of the (affine) hyperplanes $\quad H_{\alpha, j}=\left\{x \in \mathfrak{h}_{\mathbb{R}}^{*} \mid\left\langle x, \alpha^{\vee}\right\rangle=j\right\}$

in $\mathfrak{h}_{\mathbb{R}}^{*}$. The fundamental alcove is the alcove

$$
A \subseteq C \quad \text { such that } \quad 0 \in \bar{A},
$$


where $\bar{A}$ is the closure of $A$. An example is the case of type $C_{2}$, where the picture is

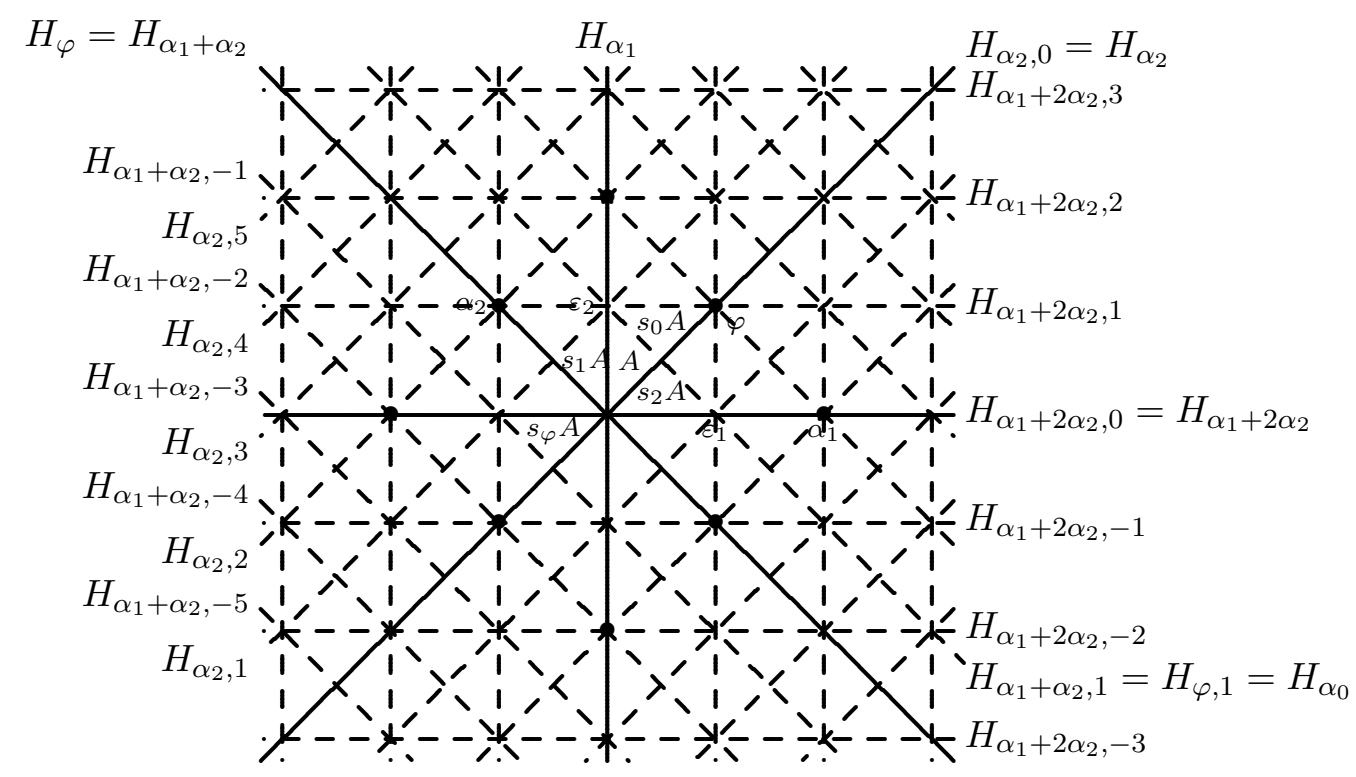

The translation in $\lambda$ is the operator $t_{\lambda}: \mathfrak{h}_{\mathbb{R}}^{*} \rightarrow \mathfrak{h}_{\mathbb{R}}^{*}$ given by

$$
t_{\lambda}(x)=\lambda+x, \quad \text { for } \lambda \in P, x \in \mathfrak{h}_{\mathbb{R}}^{*} .
$$

The reflection $s_{\alpha, k}$ in the hyperplane $H_{\alpha, k}$ is given by

$$
s_{\alpha, k}=t_{k \alpha} s_{\alpha}=s_{\alpha} t_{-k \alpha} \text {. }
$$

The extended affine Weyl group is

$$
\widetilde{W}=P \rtimes W=\left\{t_{\lambda} w \mid \lambda \in P, w \in W\right\} \quad \text { with } \quad w t_{\lambda}=t_{w \lambda} w .
$$

Denote the walls of $C$ by $H_{\alpha_{1}}, \ldots, H_{\alpha_{n}}$ and extend this indexing so that

$$
H_{\alpha_{0}}, \ldots, H_{\alpha_{n}} \quad \text { are the walls of } \quad A,
$$

the fundamental alcove. Then the affine Weyl group,

$$
W_{\text {aff }}=Q \rtimes W \text { is generated by } s_{0}, \ldots, s_{n},
$$

the reflections in the hyperplanes $H_{\alpha_{0}}, \ldots, H_{\alpha_{n}}$. Furthermore, $A$ is a fundamental region for the action of $W_{\text {aff }}$ on $\mathfrak{h}_{\mathbb{R}}^{*}$ and so there is a bijection

$$
\begin{aligned}
W_{\text {aff }} & \longrightarrow\left\{\text { alcoves in } \mathfrak{h}_{\mathbb{R}}^{*}\right\} \\
w & \longmapsto w^{-1} A .
\end{aligned}
$$

The length of $w \in \widetilde{W}$ is

$$
\ell(w)=\text { number of hyperplanes between } A \text { and } w A .
$$


The difference between $W_{\text {aff }}$ and $\widetilde{W}$ is the group

$$
\Omega=\widetilde{W} / W_{\mathrm{aff}} \cong P / Q
$$

The group $\Omega$ is the set of elements of $\widetilde{W}$ of length 0 . An element of $\Omega$ acts on the fundamental alcove $A$ by an automorphism. Its action on $A$ induces a permutation of the walls of $A$, and hence a permutation of $0,1, \ldots, n$. If $g \in \Omega$ and $g \neq 1$ let $\omega_{i}$ be the image of the origin under the action of $g$ on $A$. If $s_{j}$ denotes the reflection in the $j$ th wall of $A$ and $w_{i}$ denotes the longest element of the stabilizer $W_{\omega_{i}}$ of $\omega_{i}$ in $W$, then

$$
g s_{i} g^{-1}=s_{g(i)} \quad \text { and } \quad g w_{0} w_{i}=t_{\omega_{i}} .
$$

The group $\widetilde{W}$ acts freely on $\Omega \times \mathfrak{h}_{\mathbb{R}}^{*}\left(|\Omega|\right.$ copies of $\mathbb{R}^{n}$ tiled by alcoves) so that $g^{-1} A$ is in the same spot as $A$ except on the $g$ th "sheet" of $\Omega \times \mathfrak{h}_{\mathbb{R}}^{*}$. It is helpful to think of the elements of $\Omega$ as the deck transformations which transfer between the sheets in $\Omega \times \mathfrak{h}_{\mathbb{R}}^{*}$. Then

$$
\begin{aligned}
& \widetilde{W} \longrightarrow\left\{\text { alcoves in } \Omega \times \mathfrak{h}_{\mathbb{R}}^{*}\right\} \\
& w \longmapsto \quad w^{-1} A
\end{aligned}
$$

is a bijection. In type $C_{2}$, the two sheets in $\Omega \times \mathfrak{h}_{\mathbb{R}}^{*}$ look like

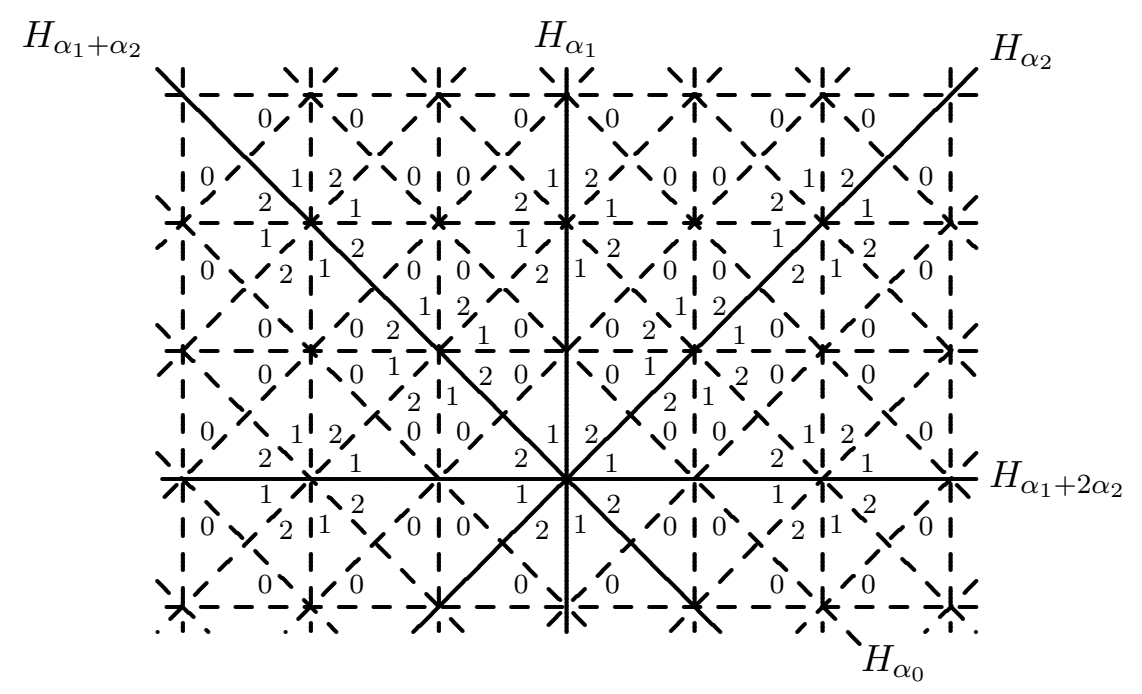


and

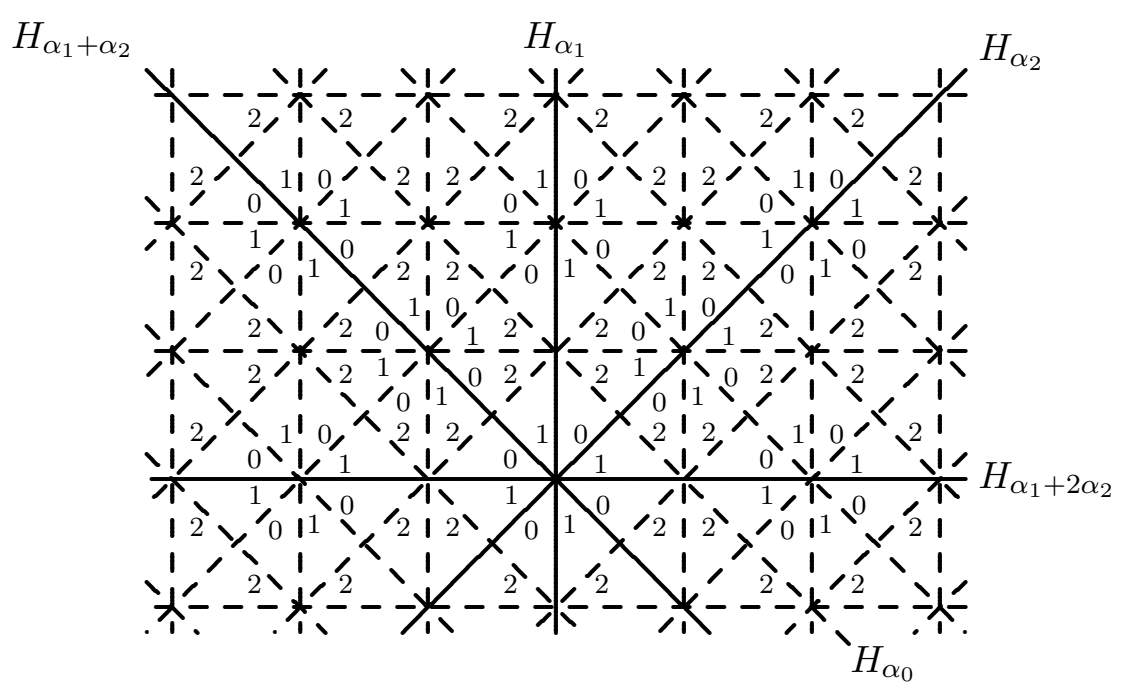

where the numbering on the walls of the alcoves is $\widetilde{W}$ equivariant so that, for $w \in \tilde{W}$, the numbering on the walls of $w A$ is the $w$ image of the numbering on the walls of $A$.

The 0-polygon is the $W$-orbit of $A$ in $\Omega \times \mathfrak{h}_{\mathbb{R}}^{*}$ and for $\lambda \in P$, the

the $\lambda$-polygon is $\lambda+W A$,

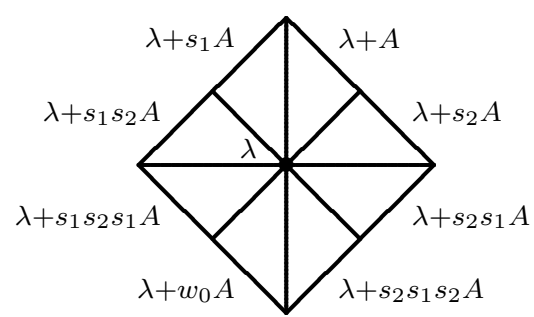

the translate of the $W$ orbit of $A$ by $\lambda$. The space $\Omega \times \mathfrak{h}_{\mathbb{R}}^{*}$ is tiled by the polygons and, via (2.11), we make identifications between $W, \widetilde{W}, P$ and their geometric counterparts in $\Omega \times \mathfrak{h}_{\mathbb{R}}^{*}$ :

$$
\widetilde{W}=\{\text { alcoves }\}, \quad W=\{\text { alcoves in the } 0 \text {-polygon }\},
$$

$$
P=\{\text { centers of polygons }\} \text {. }
$$

Define

$$
P^{+}=P \cap \bar{C} \quad \text { and } \quad P^{++}=P \cap C
$$


so that $P^{+}$is a set of representatives of the orbits of the action of $W$ on $P$. The fundamental weights are the generators $\omega_{1}, \ldots, \omega_{n}$ of the $\mathbb{Z}_{\geq 0}$-module $P^{+}$so that

$$
C=\sum_{i=1}^{n} \mathbb{R}_{\geq 0} \omega_{i}, \quad P^{+}=\sum_{i=1}^{n} \mathbb{Z}_{\geq 0} \omega_{i}, \quad \text { and } \quad P^{++}=\sum_{i=1}^{n} \mathbb{Z}_{>0} \omega_{i} .
$$

The lattice $P$ has $\mathbb{Z}$-basis $\omega_{1}, \ldots, \omega_{n}$ and the map

$$
\begin{aligned}
P^{+} & \longrightarrow P^{++} \\
\lambda & \longmapsto \rho+\lambda, \quad \text { where } \quad \rho=\omega_{1}+\ldots+\omega_{n},
\end{aligned}
$$

is a bijection. The simple coroots are $\alpha_{1}^{\vee}, \ldots, \alpha_{n}^{\vee}$ the dual basis to the fundamental weights,

$$
\left\langle\omega_{i}, \alpha_{j}^{\vee}\right\rangle=\delta_{i j}
$$

Define

$$
\overline{C^{\vee}}=\sum_{i=1}^{n} \mathbb{R}_{\leq 0} \alpha_{i}^{\vee} \quad \text { and } \quad C^{\vee}=\sum_{i=1}^{n} \mathbb{R}_{<0} \alpha_{i}^{\vee} .
$$

The dominance order is the partial order on $\mathfrak{h}_{\mathbb{R}}^{*}$ given by

$$
\mu \leq \lambda \quad \text { if } \quad \mu \in \lambda+\overline{C^{\vee}} .
$$

In type $C_{2}$ the lattice $P=\mathbb{Z} \varepsilon_{1}+\mathbb{Z} \varepsilon_{2}$ with $\left\{\varepsilon_{1}, \varepsilon_{2}\right\}$ an orthonormal basis of $\mathfrak{h}_{\mathbb{R}}^{*} \cong \mathbb{R}^{2}$ and $W=\left\{1, s_{1}, s_{2}, s_{1} s_{2}, s_{2} s_{1}, s_{1} s_{2} s_{1}, s_{2} s_{1} s_{2}, s_{1} s_{2} s_{1} s_{2}\right\}$ is the dihedral group of order 8 generated by the reflections $s_{1}$ and $s_{2}$ in the hyperplanes $H_{\alpha_{1}}$ and $H_{\alpha_{2}}$, respectively, where

$$
H_{\alpha_{1}}=\left\{x \in \mathfrak{h}_{\mathbb{R}}^{*} \mid\left\langle x, \varepsilon_{1}\right\rangle=0\right\} \quad \text { and } \quad H_{\alpha_{2}}=\left\{x \in \mathfrak{h}_{\mathbb{R}}^{*} \mid\left\langle x, \varepsilon_{2}-\varepsilon_{1}\right\rangle=0\right\} .
$$

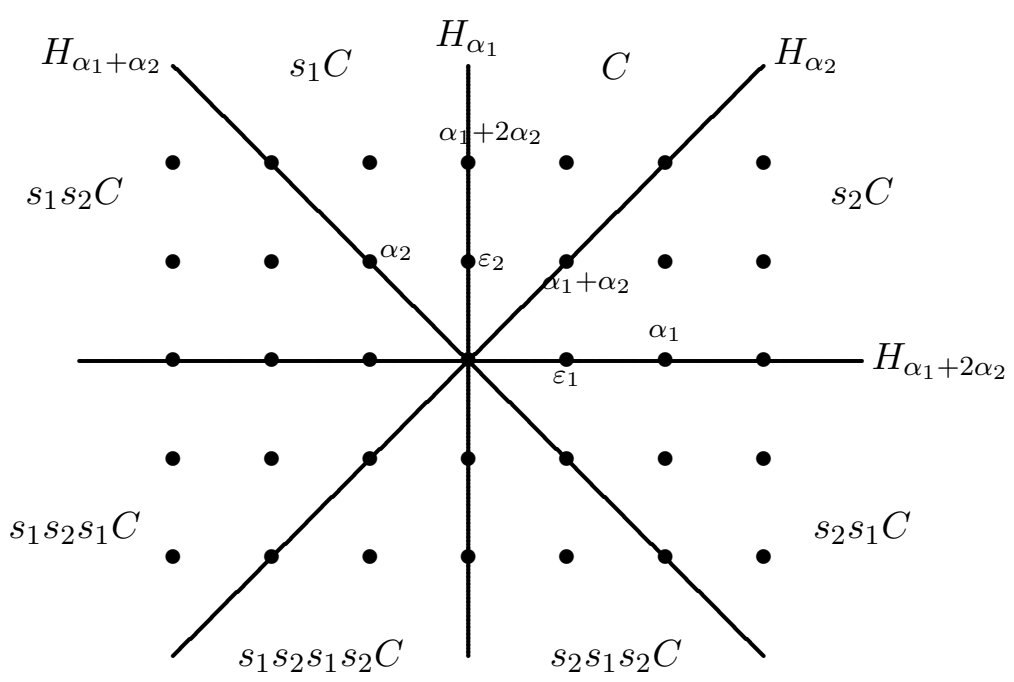




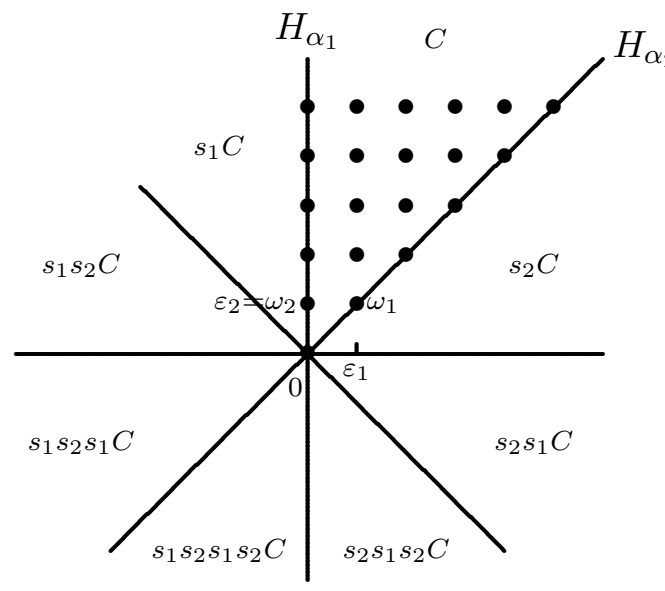

The set $P^{+}$

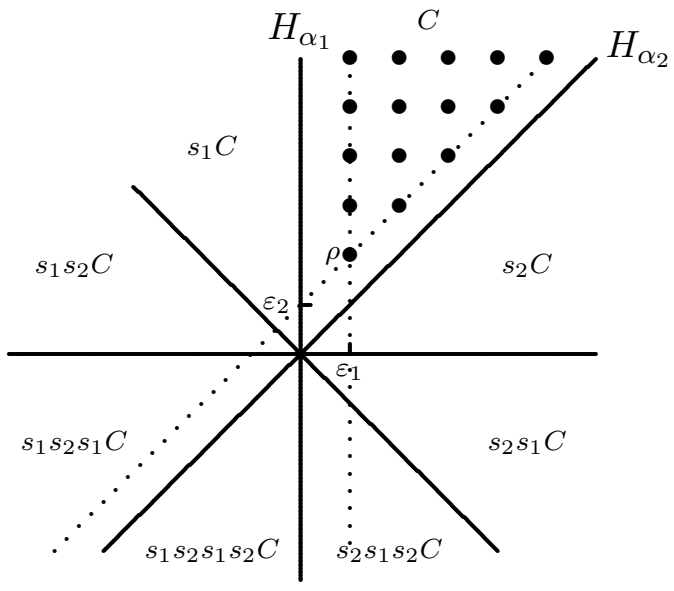

The set $P^{++}$

In this case

$$
\begin{aligned}
& \omega_{1}=\varepsilon_{1}+\varepsilon_{2}, \\
& \alpha_{1}=2 \varepsilon_{1}, \\
& \alpha_{2}=\varepsilon_{2}-\varepsilon_{1}, \\
& \alpha_{1}^{\vee}=\varepsilon_{1}, \\
& \omega_{2}=\varepsilon_{2} \text {, }
\end{aligned}
$$$$
R=\left\{ \pm \alpha_{1}, \pm \alpha_{2}, \pm\left(\alpha_{1}+\alpha_{2}\right), \pm\left(\alpha_{1}+2 \alpha_{2}\right)\right\}
$$

\section{The affine Hecke Algebra}

3.1. The alcove walk algebra. Fix notations for the Weyl group $W$, the extended affine Weyl group $\widetilde{W}$, and their action on $\Omega \times \mathfrak{h}_{\mathbb{R}}^{*}$ as in Section 2. Label the walls of the alcoves so that the fundamental alcove has walls labeled $0,1, \ldots, n$ and the labeling is $\widetilde{W}$-equivariant (see the picture in (2.12)).

The periodic orientation is the orientation of the walls of the alcoves given by (3.1) setting the positive side of $H_{\alpha, j} \quad$ to be $\quad\left\{x \in \mathfrak{h}_{\mathbb{R}}^{*} \mid\left\langle x, \alpha^{\vee}\right\rangle>j\right\}$.

This is an orientation of the walls of the alcoves such that if $\triangle$ is an alcove and $\lambda \in P$ then

the walls of $\lambda+\triangle$ have the same orientation as the walls of $\triangle$.

Let $\mathbb{K}$ be a field. Use the notations for elements of $\Omega$ as in (2.10). The alcove walk algebra is the algebra over $\mathbb{K}$ given by generators $g \in \Omega$ and

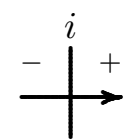

positive $i$-crossing

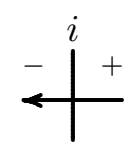

negative $i$-crossing

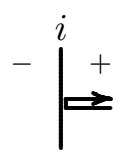

positive $i$-fold<smiles>CC(C)(C)[I-]I</smiles>

$(1 \leq i \leq n)$ negative

$i$-fold 
with relations (straightening laws)

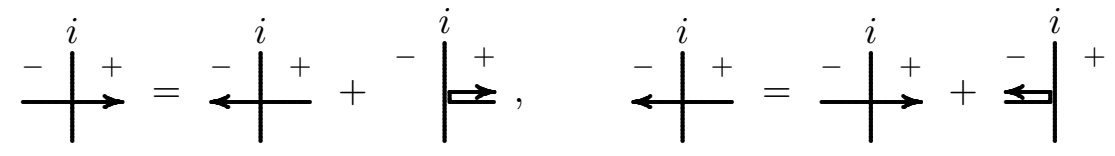

and

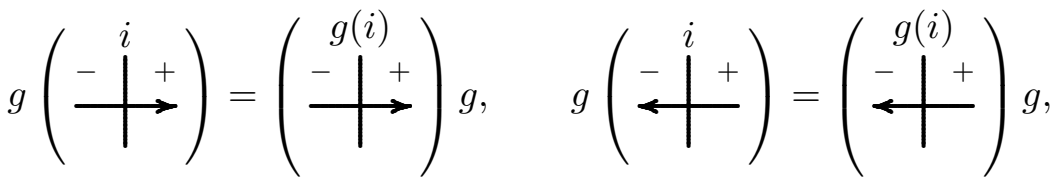

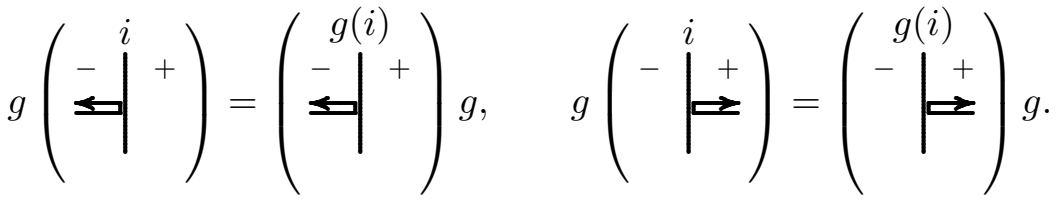

Viewing the product as concatenation each word in the generators can be represented as a sequence of arrows, with the first arrow having its head or its tail in the fundamental alcove. An alcove walk is a word in the generators such that,

(a) the tail of the first step is in the fundamental alcove $A$,

(b) at every step, the head of each arrow is in the same alcove as the tail of the next arrow.

The type of a walk $p$ is the sequence of labels on the arrows. Note that, if $w \in \widetilde{W}$ then

$$
\ell(w)=\text { length of a minimal length walk from } A \text { to } w A \text {. }
$$

For example, in type $C_{2}$,

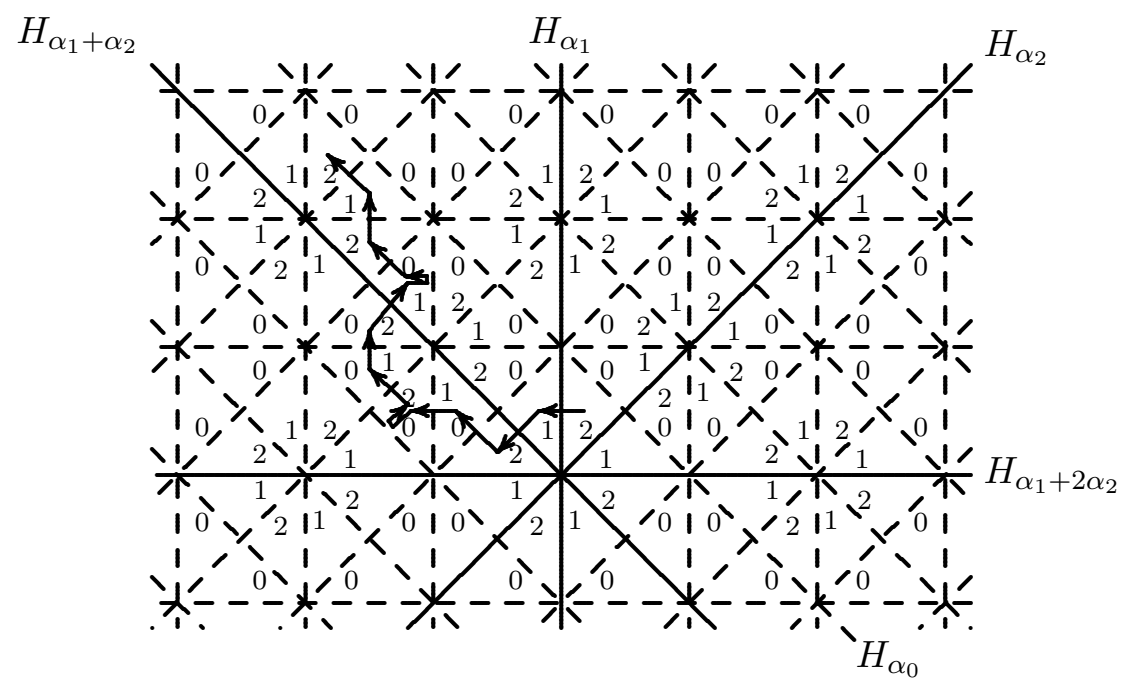


is an alcove walk $p$ of type $(1,2,0,1,0,2,1,2,1,0,1,2)$ with two folds. Using the notation

$$
\begin{array}{ll}
c_{i}^{+} \text {for a positive } i \text {-crossing, } & f_{i}^{+} \text {for a positive } i \text {-fold, } \\
c_{i}^{-} \text {for a negative } i \text {-crossing, } & f_{i}^{-} \text {for a negative } i \text {-fold, }
\end{array}
$$

the walk in the picture is $c_{1}^{-} c_{2}^{-} c_{0}^{+} c_{1}^{-} f_{0}^{+} c_{2}^{+} c_{1}^{+} c_{2}^{+} f_{1}^{-} c_{0}^{+} c_{1}^{+} c_{2}^{+}$.

The proof of the following lemma is straightforward following the scheme indicated by the example which follows.

Lemma 3.1. The set of alcove walks is a basis of the alcove walk algebra.

For example, in type $C_{2}$, a product of the generators which is not a walk is

$$
c_{1}^{-} c_{2}^{+} c_{0}^{+} c_{1}^{-} f_{0}^{-} c_{2}^{+} c_{1}^{-} c_{2}^{+} f_{1}^{-} c_{0}^{+} c_{1}^{+} c_{2}^{-},
$$

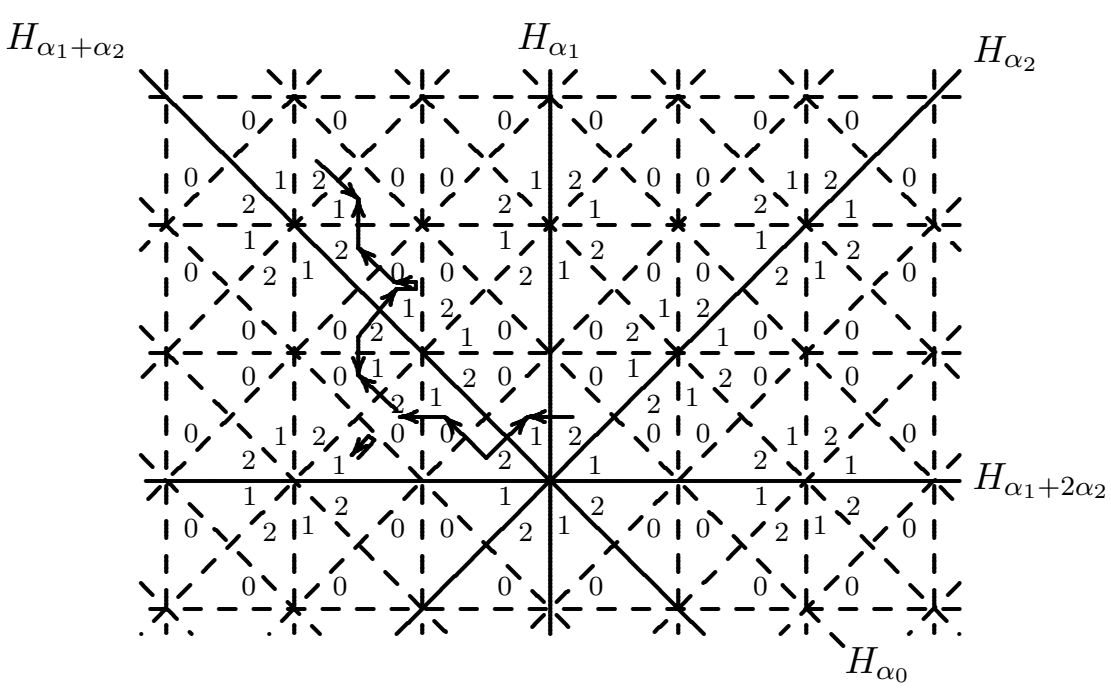

but, by first applying relations $f_{i}^{\mp}=-f_{i}^{ \pm}$and then working left to right applying the relations $c_{i}^{ \pm}=c_{i}^{\mp}+f_{i}^{ \pm}$, gives

$c_{1}^{-} c_{2}^{+} c_{0}^{+} c_{1}^{-} f_{0}^{-} c_{2}^{+} c_{1}^{-} c_{2}^{+} f_{1}^{-} c_{0}^{+} c_{1}^{+} c_{2}^{-}=$

$$
\begin{aligned}
& =-\left(c_{1}^{-} c_{2}^{+} c_{0}^{+} c_{1}^{-} f_{0}^{+} c_{2}^{+} c_{1}^{-} c_{2}^{+} f_{1}^{-} c_{0}^{+} c_{1}^{+} c_{2}^{-}\right) \\
& =-\left(c_{1}^{-}\left(c_{2}^{-}+f_{2}^{+}\right) c_{0}^{+} c_{1}^{-} f_{0}^{+} c_{2}^{+} c_{1}^{-} c_{2}^{+} f_{1}^{-} c_{0}^{+} c_{1}^{+} c_{2}^{-}\right) \\
& =-\left(c_{1}^{-}\left(c_{2}^{-}+f_{2}^{+}\right) c_{0}^{+} c_{1}^{-} f_{0}^{+} c_{2}^{+}\left(c_{1}^{+}+f_{1}^{-}\right) c_{2}^{+} f_{1}^{-} c_{0}^{+} c_{1}^{+} c_{2}^{-}\right) \\
& =-\left(c_{1}^{-}\left(c_{2}^{-}+f_{2}^{+}\right) c_{0}^{+} c_{1}^{-} f_{0}^{+} c_{2}^{+}\left(c_{1}^{+}+f_{1}^{-}\right) c_{2}^{+} f_{1}^{-} c_{0}^{+} c_{1}^{+}\left(c_{2}^{+}+f_{2}^{-}\right)\right)
\end{aligned}
$$

and every term in the expansion of this expression is an alcove walk. 
3.2. The affine Hecke algebra. Fix an invertible element $q \in \mathbb{K}$. The affine Hecke algebra $\tilde{H}$ is the quotient of the alcove walk algebra by the relations

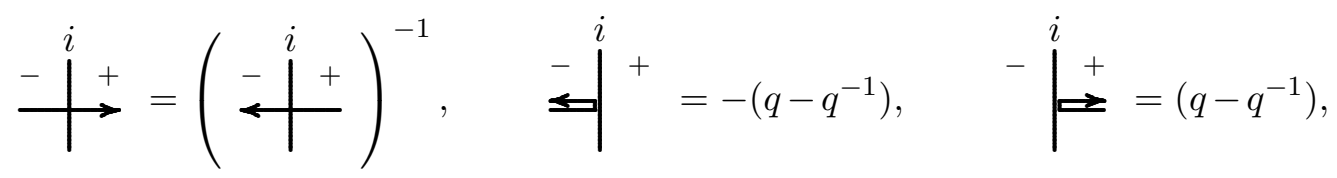

and

$$
p=p^{\prime} \quad \text { if } p \text { and } p^{\prime} \text { are nonfolded walks with } \operatorname{end}(p)=\operatorname{end}\left(p^{\prime}\right),
$$

where end $(p)$ is the final alcove of $p$. Conceptually, the affine Hecke algebra only remembers the ending alcove of a walk (and some information about the folds) and forgets how it got to its destination.

For $w \in W$ and $\lambda \in P$ define elements

$$
\begin{aligned}
T_{w^{-1}}^{-1} & =(\text { image in } \tilde{H} \text { of a minimal length alcove walk from } A \text { to } w A), \\
X^{\lambda} & =\left(\text { image in } \tilde{H} \text { of a minimal length alcove walk from } A \text { to } t_{\lambda} A\right) .
\end{aligned}
$$
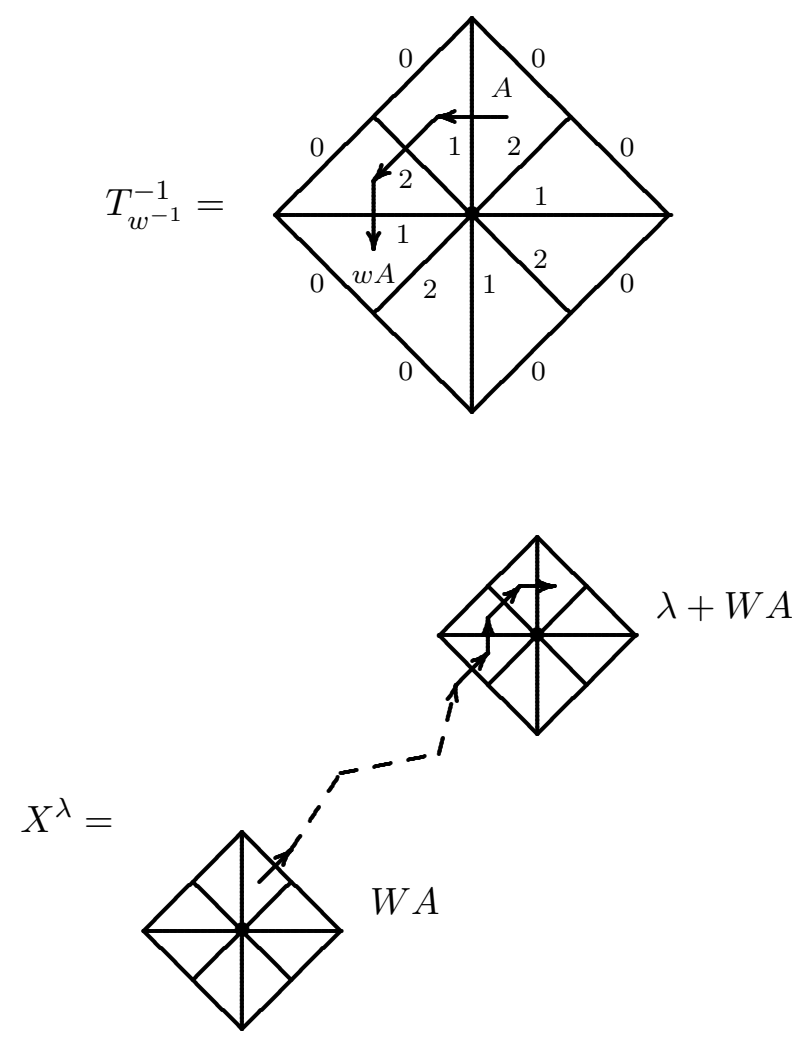

The following proposition shows that the alcove walk definition of the affine Hecke algebra coincides with the standard definition by generators and relations (see 
$[\mathrm{IM}]$ and $[\mathrm{Lu}])$. A consequence of the proposition is that

$$
\begin{array}{ll}
\text { the finite Hecke algebra, } & H=\operatorname{span}\left\{T_{w^{-1}}^{-1} \mid w \in W\right\}, \text { and } \\
\text { the Laurent polynomial ring, } & \mathbb{K}[P]=\operatorname{span}\left\{X^{\lambda} \mid \lambda \in P\right\},
\end{array}
$$

are subalgebras of $\tilde{H}$.

Proposition 3.2. Let $g \in \Omega, \lambda, \mu \in P, w \in W$ and $1 \leq i \leq n$. Let $\varphi$ be the element of $R^{+}$such that $H_{\alpha_{0}}=H_{\varphi, 1}$ is the wall of $A$ which is not a wall of $C$ and let $s_{\varphi}$ be the reflection in $H_{\varphi}$. Let $w_{0}$ be the longest element of $W$. The following identities hold in $\tilde{H}$.

(a) $X^{\lambda} X^{\mu}=X^{\lambda+\mu}=X^{\mu} X^{\lambda}$.

(b) $T_{s_{i}} T_{w}= \begin{cases}T_{s_{i} w}, & \text { if } \ell\left(s_{i} w\right)>\ell(w), \\ T_{s_{i} w}+\left(q-q^{-1}\right) T_{w}, & \text { if } \ell\left(s_{i} w\right)<\ell(w) .\end{cases}$

(c) If $\left\langle\lambda, \alpha_{i}^{\vee}\right\rangle=0$ then $T_{s_{i}} X^{\lambda}=X^{\lambda} T_{s_{i}}$.

(d) If $\left\langle\lambda, \alpha_{i}^{\vee}\right\rangle=1$ then $T_{s_{i}} X^{s_{i} \lambda} T_{s_{i}}=X^{\lambda}$.

(e) $T_{s_{i}} X^{\lambda}=X^{s_{i} \lambda} T_{s_{i}}+\left(q-q^{-1}\right) \frac{X^{\lambda}-X^{s_{i} \lambda}}{1-X^{-\alpha_{i}}}$.

(f) $T_{s_{0}} T_{s_{\varphi}}=X^{\varphi}$.

(g) $X^{\omega_{i}}=g T_{w_{0} w_{i}}$, where the action of $g$ on $A$ sends the origin to $\omega_{i}$ and $w_{i}$ is the longest element of the stabilizer $W_{\omega_{i}}$ of $\omega_{i}$ in $W$.

Proof. Use notations for alcove walks as in (3.4).

(a) If $p_{\lambda}$ is a minimal length walk from $A$ to $t_{\lambda} A$ and $p_{\mu}$ is a minimal length walk from from $A$ to $t_{\mu} A$ then

$p_{\lambda} p_{\mu}$ and $p_{\mu} p_{\lambda}$ are both nonfolded walks from $A$ to $t_{\lambda+\mu} A$.

Thus the images of $p_{\lambda} p_{\mu}$ and $p_{\mu} p_{\lambda}$ are equal in $\tilde{H}$.

(b) If $\ell\left(w s_{i}\right)>\ell(w)$ and $p_{w}$ is a minimal length walk from $A$ to $w A$ then

$$
p_{w s_{i}}=p_{w} c_{i}^{-} \text {is a minimal length walk from } A \text { to } w s_{i} A \text {. }
$$

and so $T_{s_{i} w^{-1}}^{-1}=T_{w s_{i}-1}^{-1}=T_{w^{-1}}^{-1} T_{s_{i}}^{-1}=\left(T_{s_{i}} T_{w^{-1}}\right)^{-1}$ in $\tilde{H}$. Taking inverses gives the first result, and the second follows by switching $w$ and $w s_{i}$ and using the relation $T_{s_{i}}^{-1}=T_{s_{i}}-\left(q-q^{-1}\right)$ which follows from (3.2) and (3.5).

(c) Let $p_{\lambda}$ be a minimal length alcove walk from $A$ to $t_{\lambda} A$. If $\left\langle\lambda, \alpha_{i}^{\vee}\right\rangle=0$ then $H_{\alpha_{i}}$ is a wall of $t_{\lambda} A$ and $s_{i} \lambda=\lambda$ and

$$
c_{i}^{-} p_{\lambda} c_{i}^{+} \text {is a nonfolded walk from } A \text { to } t_{\lambda} A \text {. }
$$


Thus $T_{s_{i}}^{-1} X^{\lambda} T_{s_{i}}=X^{\lambda}=X^{s_{i} \lambda}$ in $\tilde{H}$.

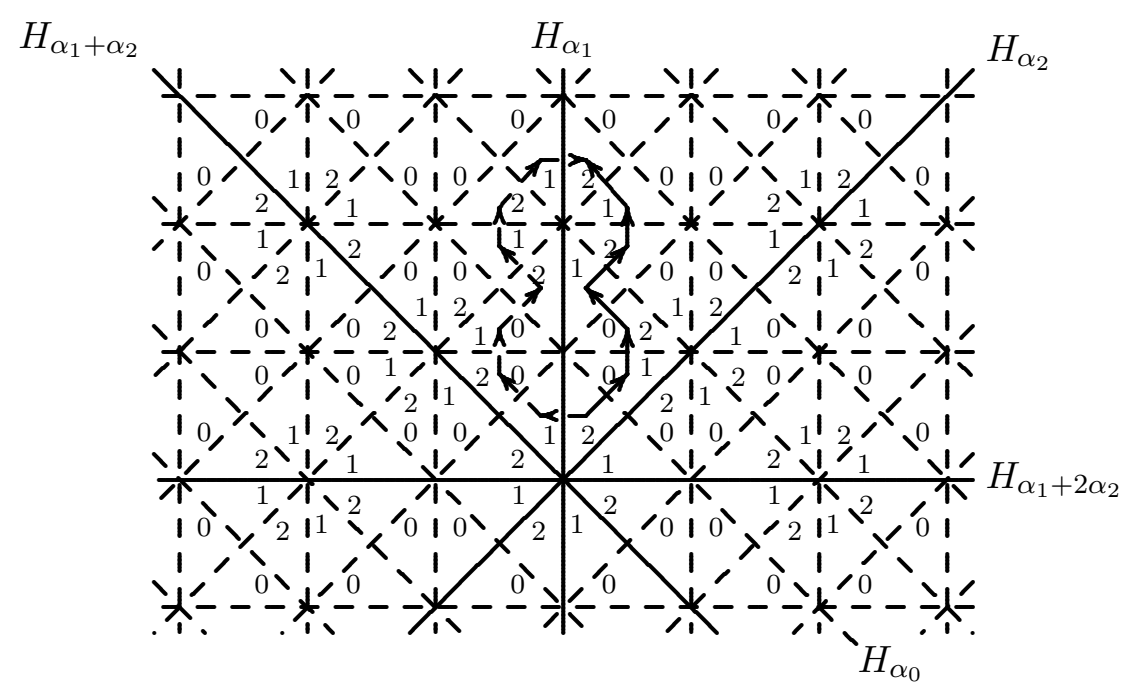

(d) Let $p_{\lambda}$ be a minimal length walk from $A$ to $t_{\lambda} A$. If $\left\langle\lambda, \alpha_{i}^{\vee}\right\rangle=1$ then there is a minimal length walk from $A$ to $t_{\lambda} A$ of the form $p_{\lambda}=p_{t_{\lambda} s_{i}} c_{i}^{+}$where $p_{t_{\lambda} s_{i}}$ is minimal length walk from $A$ to $t_{\lambda} s_{i} A$. Then

$c_{i}^{-} p_{t_{\lambda} s_{i}}$ is a minimal length walk from $A$ to $t_{s_{i} \lambda} A$.

Thus $T_{s_{i}}^{-1}\left(X^{\lambda} T_{s_{i}^{-1}}\right)=X^{s_{i} \lambda}$ in $\tilde{H}$.

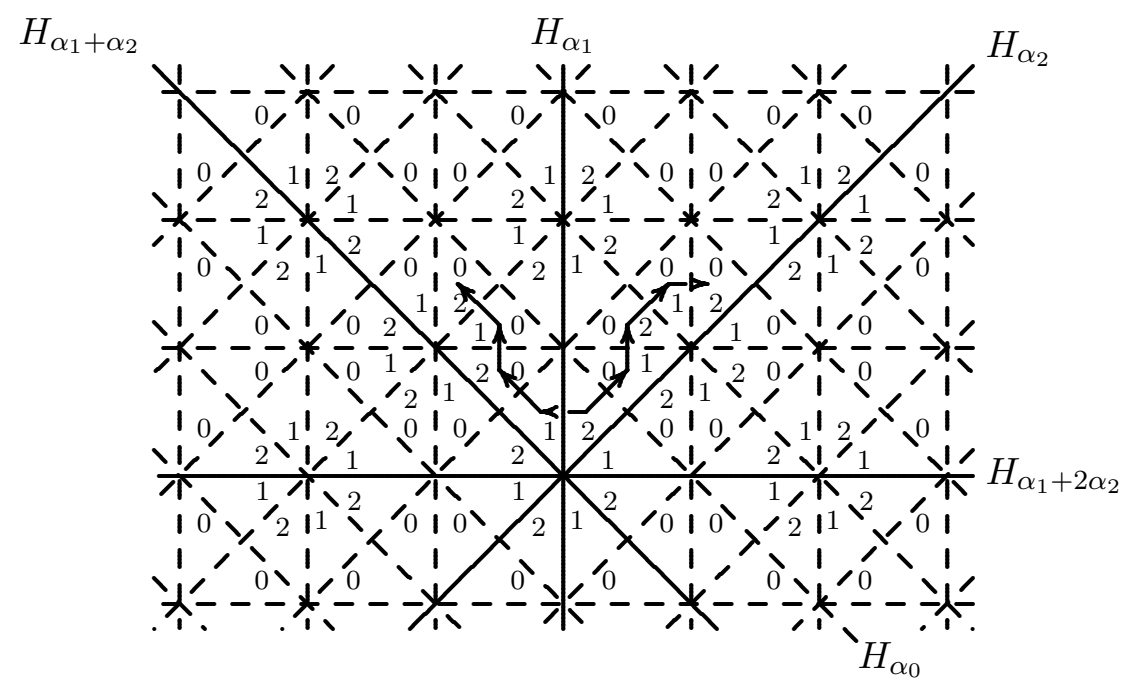

(e) Note that (c) and (d) are special cases of (e). If the statement of (e) holds for $\lambda$ then, by multiplying on the left by $X^{-s_{i} \lambda}$ and on the right by $X^{-} \lambda$, it holds 
for $-\lambda$, If the statement (e) holds for $\lambda$ and $\mu$ then it holds for $\lambda+\mu$ since

$$
\begin{aligned}
T_{s_{i}} X^{\lambda} & X^{\mu}=\left(X^{s_{i} \lambda} T_{s_{i}}+\left(q-q^{-1}\right) \frac{X^{\lambda}-X^{s_{i} \lambda}}{1-X^{-\alpha_{i}}}\right) X^{\mu} \\
& =X^{s_{i} \lambda}\left(X^{s_{i} \mu} T_{s_{i}}+\left(q-q^{-1}\right) \frac{X^{\mu}-X^{s_{i} \mu}}{1-X^{-\alpha_{i}}}\right)+\left(q-q^{-1}\right)\left(\frac{X^{\lambda}-X^{s_{i} \lambda}}{1-X^{-\alpha_{i}}}\right) X^{\mu} \\
& =X^{s_{i}(\lambda+\mu)} T_{s_{i}}+\left(q-q^{-1}\right) \frac{X^{\lambda+\mu}-X^{s_{i}(\lambda+\mu)}}{1-X^{-\alpha_{i}}} .
\end{aligned}
$$

Thus, to prove (e) it is sufficient to verify (c) and (d), which has already been done.

(f) Let $p_{s_{\varphi}}$ be a minimal length walk from $s_{\varphi} A$ to $A$, then

$$
p_{\varphi}=c_{0}^{+} p_{s_{\varphi}} \text { is a minimal length walk from } A \text { to } t_{\varphi} A \text {. }
$$

Thus $T_{0} T_{s_{\varphi}}=X^{\varphi}$ in $\tilde{H}$.

(g) If $p_{w_{0} w_{i}}$ is a minimal length walk from $w_{i} w_{0} A$ to $A$ then

$$
p_{\omega_{i}}=g p_{w_{0} w_{i}} \text { is a minimal length walk from } A \text { to } t_{\omega_{i}} A \text {. }
$$

Thus $X^{\omega_{i}}=g T_{w_{0} w_{i}}$ in $\tilde{H}$. For example, in type $C_{2}, w_{0}=s_{2} s_{1} s_{2} s_{1}$ and there is one element $g$ in $\Omega$ such that $g \neq 1$ for which $g \omega_{2}=0$ and $w_{2}=s_{1}$ so that $w_{0} w_{2}=s_{2} s_{1} s_{2}$.

The sets

$$
\left\{T_{w^{-1}}^{-1} X^{\lambda} \mid w \in W, \lambda \in P\right\} \quad \text { and } \quad\left\{X^{\mu} T_{v^{-1}}^{-1} \mid \mu \in P, v \in W\right\}
$$

are bases of $\tilde{H}$. If $p$ is an alcove walk then the weight of $p$ and the final direction of $p$ are

$$
\begin{gathered}
\operatorname{wt}(p) \in P \text { and } \varphi(p) \in W \quad \text { such that } \\
p \text { ends in the alcove } \operatorname{wt}(p)+\varphi(p) A .
\end{gathered}
$$

Let

$$
\begin{aligned}
f^{-}(p) & =(\text { number of negative folds of } p), \\
f^{+}(p) & =(\text { number of positive folds of } p), \\
f(p) & =(\text { total number of folds of } p) .
\end{aligned}
$$

The following theorem provides a combinatorial formulation of the transition matrix between the bases in (3.7). It is a $q$-version of the main result of $[\mathrm{LP}]$ and an extension of Corollary 6.1 of $[\mathrm{Sc}]$.

Theorem 3.3. Use notations as in (3.4). Let $\lambda \in P$ and $w \in W$. Fix a minimal length walk $p_{w}=c_{i_{1}}^{-} c_{i_{2}}^{-} \cdots c_{i_{r}}^{-}$from $A$ to $w A$ and a minimal length walk 
$p_{\lambda}=c_{j_{1}}^{\epsilon_{1}} \cdots c_{j_{s}}^{\epsilon_{s}}$ from $A$ to $t_{\lambda} A$. Then, with notations as in (3.8) and (3.9),

$$
T_{w^{-1}}^{-1} X^{\lambda}=\sum_{p}(-1)^{f^{-}(p)}\left(q-q^{-1}\right)^{f(p)} X^{\mathrm{wt}(p)} T_{\varphi(p)^{-1}}^{-1},
$$

where the sum is over all alcove walks $p=c_{i_{1}}^{-} \cdots c_{i_{r}}^{-} p_{j_{1}} \cdots p_{j_{s}}$ such that $p_{j_{k}}$ is either $c_{j_{k}}^{\epsilon_{k}}, c_{j_{k}}^{-\epsilon_{k}}$ or $f_{j_{k}}^{\epsilon_{k}}$.

Proof. The product $p_{w} p_{\lambda}=c_{i_{1}}^{-} c_{i_{2}}^{-} \cdots c_{i_{r}}^{-} c_{j_{1}}^{\epsilon_{1}} \cdots c_{j_{s}}^{\epsilon_{s}}$ may not necessarily be walk, but its straightening produces a sum of walks, and this decomposition gives the formula in the statement.

Remark 3.4. The initial direction $\iota(p)$ and the final direction $\varphi(p)$ of an alcove walk $p$ appear naturally in Theorem 3.3. These statistics also appear in the PieriChevalley formula in the $K$-theory of the flag variety (see $[\mathrm{PR}],[\mathrm{GR}],[\mathrm{Br}]$ and $[\mathrm{LP}])$.

Remark 3.5. In Theorem 3.3, for certain $\lambda$ the walk $p_{\lambda}$ may be chosen so that all the terms in the expansion of $T_{w^{-1}}^{-1} X^{\lambda}$ have the same sign. For example, if $\lambda$ is dominant, then $p_{\lambda}$ can be taken with all $\epsilon_{k}=+$, in which case all folds which appear in the straightening of $p_{w} p_{\lambda}$ will be positive folds and so all terms in the expansion will be positive. If $\lambda$ is antidominant then $p_{\lambda}$ can be taken with all $\epsilon_{k}=-$ and all terms in the expansion will be negative. This fact gives positivity results for products in the cohomology and the $K$-theory of the flag variety (see $[\mathrm{PR}],[\mathrm{Br}])$.

Remark 3.6. The affine Hecke algebra $\tilde{H}$ has basis $\left\{X^{\lambda} T_{w^{-1}}^{-1} \mid \lambda \in P, w \in W\right\}$ in bijection with the alcoves in $\Omega \times \mathfrak{h}_{\mathbb{R}}^{*}$, where $X^{\lambda} T_{w^{-1}}^{-1}$ is the image in $\tilde{H}$ of a minimal length alcove walk from $A$ to the alcove $\lambda+w A$. Changing the orientation of the walls of the alcoves changes the resulting basis in the affine Hecke algebra $\tilde{H}$. The orientation in (3.1) is the one such that

$$
\text { the most negative point is }-\infty \rho \text {, deep in the chamber } w_{0} C \text {. }
$$

Another standard orientation is where

$$
\text { the most negative point is the center of the fundamental alcove } A \text {. }
$$

Using the orientation of the walls given by (3.11) produces the basis commonly denoted $\left\{T_{w} \mid w \in \widetilde{W}\right\}$ by taking $T_{w}$ to be the image in $\tilde{H}$ of a minimal length alcove walk from $A$ to $w^{-1} A$. Since $T_{i}^{-1}=T_{i}-\left(q-q^{-1}\right)$ the transition matrix between the basis $\left\{X^{\lambda} T_{w^{-1}}^{-1} \mid \lambda \in P, w \in W\right\}$ and the basis $\left\{T_{w} \mid w \in \widetilde{W}\right\}$ is triangular. 


\section{Polynomials And Symmetric Functions}

Recall, from (3.6), that the finite Hecke algebra is the subalgebra of the affine Hecke algebra $\tilde{H}$ given by

$$
H=\operatorname{span}\left\{T_{w^{-1}}^{-1} \mid w \in W\right\} .
$$

Let $\mathbf{1}_{0}$ be the element of $H$ given by

$$
\mathbf{1}_{0}^{2}=\mathbf{1}_{0} \quad \text { and } \quad T_{w^{-1}}^{-1} \mathbf{1}_{0}=q^{-\ell(w)} \mathbf{1}_{0},
$$

for $w \in W$. Two explicit formulas for $\mathbf{1}_{0}$ are

$$
\mathbf{1}_{0}=\frac{1}{W_{0}\left(q^{-2}\right)} \sum_{w \in W} q^{-\ell(w)} T_{w^{-1}}^{-1}=\frac{1}{W_{0}\left(q^{2}\right)} \sum_{w \in W} q^{\ell(w)} T_{w}
$$

where $W_{0}(t)=\sum_{w \in W} t^{\ell(w)}$ is the Poincaré polynomial of $W$.

As observed in (3.6), $\mathbb{K}[P]=\operatorname{span}\left\{X^{\lambda} \mid \lambda \in P\right\}$ is a subalgebra of $\tilde{H}$. The vector space $\mathbb{K}[P]$ also sits inside $\tilde{H}$ in a different way. Since $\left\{X^{\lambda} \mathbf{1}_{0} \mid \lambda \in P\right\}$ is a basis of $\tilde{H} \mathbf{1}_{0}$ there is

$$
\text { a vector space isomorphism } \begin{aligned}
\mathbb{K}[P] & \longrightarrow \tilde{H} \mathbf{1}_{0} \\
f & \longmapsto f \mathbf{1}_{0} .
\end{aligned}
$$

The ring of symmetric functions is

$$
\mathbb{K}[P]^{W}=\{f \in \mathbb{K}[P] \mid w f=f \text { for all } w \in W\} .
$$

By a theorem of Bernstein (see [NR, Theorem 1.4]) this subalgebra of $\tilde{H}$ is the center of $\tilde{H}$,

$$
\mathbb{K}[P]^{W}=Z(\tilde{H}) .
$$

The spherical Hecke algebra is the ring $\mathbf{1}_{0} X^{\lambda} \mathbf{1}_{0}$ and the restriction of the map (4.2) to $Z(\tilde{H})$ is the Satake isomorphism of the following theorem.

Theorem 4.1. Let $\mathbf{1}_{0}$ and $\mathbb{K}[P]^{W}$ be as in (4.1) and (4.3), respectively. Then

$$
\begin{aligned}
\mathbb{K}[P]^{W}=Z(\tilde{H}) & \longrightarrow \mathbf{1}_{0} \tilde{H} \mathbf{1}_{0} \quad \text { is a } \mathbb{K} \text {-algebra isomorphism. } \\
\quad & \longmapsto f \mathbf{1}_{0}
\end{aligned}
$$

Proof. The map is a well defined homomorphism since, if $f, f_{1}, f_{2} \in Z(\tilde{H})$, then $f \mathbf{1}_{0}=f \mathbf{1}_{0}^{2}=\mathbf{1}_{0} f \mathbf{1}_{0}$ and $f_{1} f_{2} \mathbf{1}_{0}=f_{1} f_{2} \mathbf{1}_{0}^{2}=f_{1} \mathbf{1}_{0} f_{2} \mathbf{1}_{0}$. 
Suppose that $\left\langle\lambda, \alpha_{i}^{\vee}\right\rangle>0$ so that $\lambda$ is on the positive side of $H_{\alpha_{i}}$. Then, by Proposition 3.2e,

$$
\begin{aligned}
\mathbf{1}_{0} X^{s_{i} \lambda} \mathbf{1}_{0} & =q^{-1} \mathbf{1}_{0} T_{s_{i}} X^{\lambda} \mathbf{1}_{0} \\
& =q^{-1} \mathbf{1}_{0} X^{\lambda} T_{s_{i}} \mathbf{1}_{0}-q^{-1}\left(q-q^{-1}\right) \mathbf{1}_{0}\left(X^{s_{i} \lambda+\alpha_{i}}+\cdots+X^{\lambda-\alpha_{i}}+X^{\lambda}\right) \mathbf{1}_{0} \\
& =\mathbf{1}_{0} X^{\lambda} \mathbf{1}_{0}-\left(1-q^{-2}\right) \mathbf{1}_{0}\left(X^{s_{i} \lambda+\alpha_{i}}+\cdots+X^{\lambda-\alpha_{i}}+X^{\lambda}\right) \mathbf{1}_{0} .
\end{aligned}
$$

so that

$$
\mathbf{1}_{0} X^{s_{i} \lambda} \mathbf{1}_{0}=q^{-2} \mathbf{1}_{0} X^{\lambda} \mathbf{1}_{0}-\left(1-q^{-2}\right) \mathbf{1}_{0}\left(X^{s_{i} \lambda+\alpha_{i}}+\cdots+X^{\lambda-\alpha_{i}}\right) \mathbf{1}_{0}
$$

or, equivalently,

$$
\mathbf{1}_{0}\left(X^{s_{i} \lambda}+\cdots+X^{\lambda-\alpha_{i}}\right) \mathbf{1}_{0}=q^{-2} \mathbf{1}_{0}\left(X^{s_{i} \lambda+\alpha_{i}}+\cdots+X^{\lambda}\right) \mathbf{1}_{0} .
$$

From the relation in (4.5),

$$
\begin{array}{r}
\mathbf{1}_{0} X^{s_{i} \lambda} \mathbf{1}_{0}-\mathbf{1}_{0} X^{s_{i} \lambda+\alpha_{i}} \mathbf{1}_{0}=q^{-2} \mathbf{1}_{0} X^{\lambda} \mathbf{1}_{0}-\left(1-q^{-2}\right) \mathbf{1}_{0}\left(X^{s_{i} \lambda+\alpha_{i}}+\cdots+X^{\lambda-\alpha_{i}}\right) \mathbf{1}_{0} \\
-q^{-2} \mathbf{1}_{0} X^{\lambda-\alpha_{i}} \mathbf{1}_{0}+\left(1-q^{-2}\right) \mathbf{1}_{0}\left(X^{s_{i} \lambda+2 \alpha_{i}}+\cdots+X^{\lambda-2 \alpha_{i}}\right) \mathbf{1}_{0}
\end{array}
$$

so that

$$
\mathbf{1}_{0} X^{s_{i} \lambda} \mathbf{1}_{0}=q^{-2} \mathbf{1}_{0} X^{\lambda} \mathbf{1}_{0}+q^{-2} \mathbf{1}_{0} X^{s_{i} \lambda+\alpha_{i}} \mathbf{1}_{0}-\mathbf{1}_{0} X^{\lambda-\alpha_{i}} \mathbf{1}_{0} .
$$

It follows from these relations that any element of $\mathbf{1}_{0} \tilde{H} \mathbf{1}_{0}$ can, inductively, be written as a linear combination of the elements $\mathbf{1}_{0} X^{\lambda} \mathbf{1}_{0}, \lambda \in P^{+}$. Using Theorem 3.3 to expand $\mathbf{1}_{0} X^{\lambda}$ in terms of the basis $\left\{X^{\mu} T_{v^{-1}}^{-1} \mid \mu \in P, v \in W\right\}$ produces

$$
\mathbf{1}_{0} X^{\lambda}=X^{w_{0} \lambda} T_{w_{0}}^{-1}+\sum_{\mu>w_{0} \lambda} d_{\mu, v} X^{\mu} T_{v^{-1}}^{-1},
$$

and, since these leading terms are all different (as $\lambda$ runs over $P^{+}$), it follows that

$$
\mathbf{1}_{0} \tilde{H} \mathbf{1}_{0} \quad \text { has basis } \quad\left\{\mathbf{1}_{0} X^{\lambda} \mathbf{1}_{0} \mid \lambda \in P^{+}\right\} .
$$

The orbit sums

$$
m_{\lambda}=\sum_{\gamma \in W \lambda} X^{\gamma}, \quad \lambda \in P^{+},
$$

form a basis of $\mathbb{K}[P]^{W}$. The relation in Proposition 3.2e implies that, if $f \in \mathbb{K}[P]^{W}$ then $T_{w} f=f T_{w}$ for all $w \in W$, and so the $m_{\lambda} \mathbf{1}_{0}=\mathbf{1}_{0} m_{\lambda} \mathbf{1}_{0}$ are in $\mathbf{1}_{0} \tilde{H} \mathbf{1}_{0}$. Viewing these in terms of the basis $\left\{X^{\mu} T_{v^{-1}}^{-1} \mid \mu \in P, v \in W\right\}$ of $\tilde{H}$ one sees that the $m_{\lambda} \mathbf{1}_{0}, \lambda \in P^{+}$, are linearly independent and so

$$
\mathbf{1}_{0} \tilde{H} \mathbf{1}_{0} \text { has basis }\left\{m_{\lambda} \mathbf{1}_{0} \mid \lambda \in P^{+}\right\} .
$$

The point is that the transition matrix between the basis in (4.8) and the basis in (4.10) is triangular. 
4.1. Hall-Littlewood polynomials. For $\mu \in P$ let $W_{\mu}=\operatorname{Stab}(\mu)$ be the stabilizer of $\mu$. The Poincaré polynomial of $W_{\mu}$ is

$$
W_{\mu}(t)=\sum_{w \in W_{\mu}} t^{\ell(w)}
$$

For $\mu \in P$, the Hall-Littlewood polynomial or Macdonald spherical function $P_{\mu}(X ; t)$ is the element of $\mathbb{K}[P]^{W}$ defined by

$$
P_{\mu}\left(X ; q^{-2}\right) \mathbf{1}_{0}=\left(\sum_{w \in W^{\mu}} q^{-\ell(w)} T_{w^{-1}}^{-1}\right) X^{\mu} \mathbf{1}_{0}
$$

where $W^{\mu}$ is a set of minimal length coset representatives for the cosets in $W / W_{\mu}$. Since every element $w \in W$ has a unique expression $w=u v$ with $u \in W^{\mu}$ and $v \in W_{\mu}$,

$$
\begin{aligned}
& \sum_{w \in W^{\mu}} q^{-\ell(w)} T_{w^{-1}}^{-1} X^{\mu} \mathbf{1}_{0}=\frac{1}{W_{\mu}\left(q^{-2}\right)}\left(\sum_{u \in W^{\mu}} q^{-\ell(u)} T_{u^{-1}}^{-1}\right) X^{\mu}\left(\sum_{v \in W_{\mu}} q^{-\ell(v)} T_{v^{-1}}^{-1}\right) \mathbf{1}_{0} \\
& =\frac{1}{W_{\mu}\left(q^{-2}\right)}\left(\sum_{u \in W^{\mu}} q^{-\ell(u)} T_{u^{-1}}^{-1}\right)\left(\sum_{v \in W_{\mu}} q^{-\ell(v)} T_{v^{-1}}^{-1}\right) X^{\mu} \mathbf{1}_{0}=\frac{W_{0}\left(q^{-2}\right)}{W_{\mu}\left(q^{-2}\right)} \mathbf{1}_{0} X^{\mu} \mathbf{1}_{0},
\end{aligned}
$$

and hence

$$
P_{\mu}\left(X, q^{-2}\right) \mathbf{1}_{0} \quad \text { is exactly } \quad \mathbf{1}_{0} X^{\mu} \mathbf{1}_{0} \quad \text { except normalized }
$$

so that the coefficient of $X^{\mu} \mathbf{1}_{0}$ is 1 .

Macdonald's formula for the spherical functions on a $p$-adic group [Mac1, Theorem 4.1.2] is

$$
P_{\mu}\left(X ; q^{-2}\right)=\frac{1}{W_{\mu}\left(q^{-2}\right)} \sum_{w \in W} w\left(X^{\mu} \prod_{\alpha \in R^{+}} \frac{1-q^{-2} X^{-\alpha}}{1-X^{-\alpha}}\right) .
$$

See [NR, Theorem 2.9a] for a proof in this context.

The following theorem gives additional formulas for $P_{\lambda}\left(X ; q^{-2}\right)$.

(4.14) A positively folded alcove walk is an alcove walk with no negative folds.

In the following theorem we shall consider alcove walks which do not necessarily begin at $A$. This is the natural way to account for the sum over $W^{\lambda}$ which appears in the definition of $P_{\lambda}$ in (4.12). The type of $p$ is the sequence of labels of the folds and the wall crossings of $p$.

Theorem 4.2. For $\lambda \in P^{+}$let $t_{\lambda} \in \widetilde{W}$ be the translation in $\lambda$ and let $n_{\lambda}$ be the maximal length element in the double coset $W t_{\lambda} W$. 
(a) [Sc, Theorem 1.1] Let $\lambda \in P^{+}$and fix a minimal length walk $p_{\lambda}=c_{i_{1}}^{+} \cdots c_{i_{\ell}}^{+}$ from $A$ to $\lambda+A$. Let

$$
B_{q}\left(p_{\lambda}\right)=\left\{\begin{array}{l}
\text { positively folded alcove walks of } \\
\text { type }\left(i_{1}, \ldots, i_{\ell}\right) \text { which begin at } w A
\end{array} \mid w \in W^{\lambda}\right\}
$$

Then

$$
P_{\lambda}\left(X ; q^{-2}\right)=\sum_{p \in B_{q}\left(p_{\lambda}\right)} q^{-(\ell(\iota(p))+\ell(\varphi(p))-f(p))}\left(1-q^{-2}\right)^{f(p)} X^{\mathrm{wt}(p)},
$$

where $\iota(p)$ is the alcove where $p$ begins, $\operatorname{wt}(p)+\varphi(p) A$ is the alcove where $p$ ends, and $f(p)$ is the number of folds in $p$.

(b) Let $\lambda \in P^{+}$. Then

$$
q^{\ell\left(w_{0}\right)} W_{0}\left(q^{-2}\right) P_{\lambda} \mathbf{1}_{0}=\sum_{x \in W t_{\lambda} W} q^{\ell(x)-\ell\left(n_{\lambda}\right)} T_{x}
$$

Proof. (a) The proof is accomplished by using Theorem 3.3 to expand the sum in (4.12). Since all crossings in the walk $p_{\lambda}$ are positive crossings Theorem 3.3 gives

$$
\left(\sum_{w \in W^{\lambda}} q^{-\ell(w)} T_{w^{-1}}^{-1}\right) X^{\lambda}=\sum_{w \in W^{\lambda}} q^{-\ell(w)} \sum_{\substack{p \in B_{q}\left(p_{\lambda}\right) \\ \iota(p)=w}}\left(q-q^{-1}\right)^{f(p)} X^{\mathrm{wt}(p)} T_{\varphi(p)}^{-1}
$$

Hence

$$
P_{\lambda} \mathbf{1}_{0}=\sum_{p \in B_{q}(\lambda)} q^{-\ell(\iota(p))}\left(q-q^{-1}\right)^{f(p)} X^{\mathrm{wt}(p)} q^{-\ell(\varphi(p))} \mathbf{1}_{0}
$$

(b) Let $\lambda \in P^{+}$. Let $W_{\lambda}=\operatorname{Stab}(\lambda)$ and let $w_{0}$ and $w_{\lambda}$ be the maximal length elements in $W$ and $W_{\lambda}$, respectively. Let $m_{\lambda}$ and $n_{\lambda}$ be the minimal and maximal length elements respectively in the double coset $W t_{\lambda} W$. If $\lambda=2 \omega_{2}$ in type $C_{2}$, then $W_{\lambda}=\left\{1, s_{1}\right\}, w_{\lambda}=s_{1}, w_{0}=s_{1} s_{2} s_{1} s_{2}, \ell\left(t_{\lambda}\right)=6, \ell\left(m_{\lambda}\right)=3$, and $\ell\left(n_{\lambda}\right)=10$. Labeling the alcove $w A$ by the element $w$, the 32 alcoves $w A$ with $w \in W t_{\lambda} W$ 
make up the four shaded diamonds.

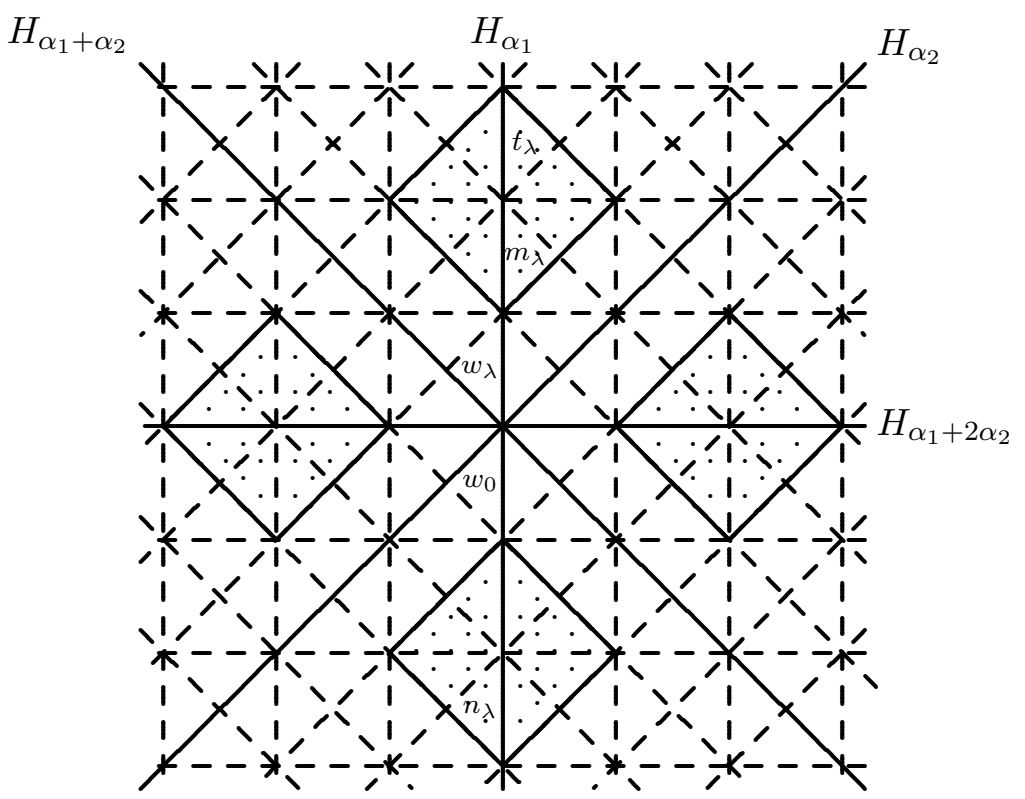

Then

$$
\begin{aligned}
q^{\ell\left(w_{0}\right)} W_{0}\left(q^{-2}\right) P_{\lambda} \mathbf{1}_{0}=q^{\ell\left(w_{0}\right)} W_{0}\left(q^{-2}\right) q^{-2 \ell\left(w_{0}\right)+2 \ell\left(w_{\lambda}\right)}\left(\sum_{u \in W^{\lambda}} q^{\ell(u)} T_{u}\right) X^{\lambda} \mathbf{1}_{0} \\
=q^{\ell\left(w_{0}\right)} q^{-2 \ell\left(w_{0}\right)} W_{0}\left(q^{2}\right) q^{-2 \ell\left(w_{0}\right)+2 \ell\left(w_{\lambda}\right)}\left(\sum_{u \in W^{\lambda}} q^{\ell(u)} T_{u}\right) T_{m_{\lambda}} T_{w_{0} w_{\lambda}} \mathbf{1}_{0} \\
=q^{-3 \ell\left(w_{0}+2 \ell\left(w_{\lambda}\right)\right.}\left(\sum_{u \in W^{\lambda}} q^{\ell(u)} T_{u}\right) T_{m_{\lambda}} q^{\ell\left(w_{0}\right)-\ell\left(w_{\lambda}\right)}\left(\sum_{w \in W} q^{\ell(w)} T_{w}\right) \\
=q^{-2 \ell\left(w_{0}\right)+\ell\left(w_{\lambda}\right)}\left(\sum_{u \in W^{\lambda}} q^{\ell(u)+\ell(w)} T_{u m_{\lambda} w}\right) \\
=q^{-2 \ell\left(w_{0}\right)+\ell\left(w_{\lambda}\right)}\left(\sum_{x \in W t_{\lambda} W} q^{\ell(x)-\ell\left(m_{\lambda}\right)} T_{x}\right)
\end{aligned}
$$

and the result follows from the identity $\ell\left(n_{\lambda}\right)=\ell\left(w_{0}\right)+\ell\left(t_{\lambda}\right)=\ell\left(w_{0}\right)+\ell\left(w_{0}\right)-$ $\ell\left(w_{\lambda}\right)+\ell\left(m_{\lambda}\right)$. 
Remark 4.3. The set $B_{q}\left(p_{\lambda}\right)$ appearing in Theorem $4.2 \mathrm{a}$ is heavily dependent on the choice of $p_{\lambda}$. One way to make this seem less dependent on this choice is as follows. For convenience assume that $\lambda$ is regular (not on any wall). Similar definitions can be made in the general case. Consider the region

$$
[\lambda]=\left\{x=\sum_{i} x_{i} \omega_{i} \mid 0 \leq x_{i} \leq \lambda_{i}\right\}, \quad \text { where } \quad \lambda=\sum_{i} \lambda_{i} \omega_{i} .
$$

This region is a union of alcoves and any minimal length walk $p_{\lambda}$ from $A$ to $\lambda+w_{0} A$ lies in $[\lambda]$. Foldings of the walk $p_{\lambda}$ are then produced by folding the region $[\lambda]$ along the "creases" formed by the hyperplanes. This process produces a bijection between the paths in $B_{q}\left(p_{\lambda}\right)$ and the set $B_{q}([\lambda])$ of "positively folded foldings" of the region $[\lambda]$, and the set $B_{q}([\lambda])$ does not depend the choice of an initial path. The moral is that the best way to forget the choice of the initial path $p_{\lambda}$ is to remember all the possible initial paths all at once. This translation of foldings was explained to me by J. Ramagge in Fall 2000.

Remark 4.4. Let $B_{q}\left(p_{\lambda}\right)$ be as in Theorem $4.2 \mathrm{a}$ and let $p \in B_{q}\left(p_{\lambda}\right)$. Suppose that $p$ has $f$ folds. For $0 \leq i \leq f$, let $p^{(i)}$ be the positively folded walk in $B_{q}\left(p_{\lambda}\right)$ which coincides with $p$ up to the $i$ th fold and is nonfolded thereafter. Then $p^{(0)}, \ldots, p^{(f)}$ is a sequence of positively folded walks such that

$$
p^{(f)}=p, \quad \iota\left(p^{(i)}\right)=\iota(p), \quad \varphi\left(p^{(0)}\right)=\iota(p), \quad \text { and } \quad \varphi\left(p^{(i)}\right)=s_{\alpha} \varphi\left(p^{(i-1)}\right)
$$

if the $i$ th fold is on the hyperplane $H_{\alpha, j}$. Since $\varphi\left(p^{(i-1)}\right)>\varphi\left(p^{(i)}\right)$ and

$$
\begin{aligned}
& (-1)^{\ell\left(\varphi\left(p^{(i)}\right)\right)}=(-1)^{\ell\left(s_{\alpha}\right)}(-1)^{\varphi\left(p^{(i-1)}\right)}=(-1)(-1)^{\varphi\left(p^{(i-1)}\right)}, \\
& \ell\left(\varphi\left(p^{(i-1)}\right)\right)-\ell\left(\varphi\left(p^{(i)}\right)\right)-1 \text { is an even integer } \geq 0 . \text { It follows that } \\
& \ell(\iota(p))+\ell(\varphi(p))-f(p) \\
& =\ell(\iota(p))-\ell\left(\varphi\left(p^{(1)}\right)\right)-1 \\
& \quad+\ell\left(\varphi\left(p^{(1)}\right)\right)-\ell\left(\varphi\left(p^{(2)}\right)\right)-1 \\
& \quad+\ell\left(\varphi\left(p^{(2)}\right)\right)-\ell\left(\varphi\left(p^{(3)}\right)\right)-1 \\
& \quad+\cdots+\ell\left(\varphi\left(p^{(f-1)}\right)\right)-\ell\left(\varphi\left(p^{(f)}\right)\right)-1+2 \ell(\varphi(p))
\end{aligned}
$$

is an even integer $\geq 0$. This proves that $f(p) \leq \ell(\iota(p))-\ell(\varphi(p))$ and that $P_{\lambda}\left(X ; q^{-2}\right)$ really is a polynomial in the variable $q^{-2}$.

4.2. Demazure operators. The group $W$ acts on $\mathbb{K}[P]=\operatorname{span}\left\{X^{\lambda} \mid \lambda \in P\right\}$ by

$$
w X^{\lambda}=X^{w \lambda}, \quad \text { for } w \in W, \lambda \in P .
$$

For each $1 \leq i \leq n$, define Demazure operators

$$
\Delta_{i}: \mathbb{K}[P] \longrightarrow \mathbb{K}[P] \quad \text { and } \quad \tilde{\Delta}_{i}: \mathbb{K}[P] \longrightarrow \mathbb{K}[P]
$$


by

$$
\Delta_{i} f=\frac{1}{1-X^{-\alpha_{i}}}\left(1-s_{i}\right) f \quad \text { and } \quad \tilde{\Delta}_{i} f=\frac{1}{X^{\alpha_{i}}-1}\left(X^{\alpha_{i}}-s_{i}\right) f,
$$

respectively.

Via the isomorphism in (4.2), the vector space $\mathbb{K}[P]$ is an $\tilde{H}$-module. Let

$$
C_{i}=q^{-2}+q^{-1} T_{s_{i}}=1+q^{-1} T_{s_{i}}^{-1}=\left(1+q^{-2}\right) \mathbf{1}_{i},
$$

where $\mathbf{1}_{i}$ is the element of $H$ such that $\mathbf{1}_{i}^{2}=\mathbf{1}_{i}$ and $T_{s_{i}}^{-1} \mathbf{1}_{i}=q^{-1} \mathbf{1}_{i}$. The element $\mathbf{1}_{i}$ is the rank 1 version of the element $\mathbf{1}_{0}$ in (4.1).

The following proposition shows that, at $q^{-2}=0$, the action of $C_{i}$ on $\mathbb{K}[P]$ is the Demazure operator $\tilde{\Delta}_{i}$. In geometry, the Demazure operators arise naturally as push-pull operators on the K-theory of the flag variety (see [PR, Proposition]).

Proposition 4.5. Let $\rho=\omega_{1}+\cdots+\omega_{n}$ as in (2.16). As operators on $\mathbb{K}[P]$,

(a) $\tilde{\Delta}_{i}=X^{-\rho} \Delta_{i} X^{\rho}=\Delta_{i}+s_{i}$,

(b) $C_{i}=\left(1-q^{-2}\right) \Delta_{i}+\left(s_{i}+q^{-2}\right)=\left(1+s_{i}\right)\left(\frac{1-q^{-2} X^{-\alpha_{i}}}{1-X^{-\alpha_{i}}}\right)$,

Proof. (a) Let $\lambda \in P$. Since $s_{i} \rho=\rho-\left\langle\rho, \alpha_{i}^{\vee}\right\rangle \alpha_{i}=\rho-\alpha_{i}$,

$$
\left(X^{-\rho} \Delta_{i} X^{\rho}\right)\left(X^{\lambda}\right)=X^{-\rho} \frac{X^{\lambda+\rho}-X^{s_{i} \lambda+\rho-\alpha_{i}}}{1-X^{-\alpha_{i}}}=\frac{X^{\lambda}-X^{s_{i} \lambda-\alpha_{i}}}{1-X^{-\alpha_{i}}}=\tilde{\Delta}_{i}\left(X^{\lambda}\right),
$$

and, as operators,

$$
\Delta_{i}+s_{i}=\frac{1}{1-X^{-\alpha_{i}}}\left(1-s_{i}\right)+s_{i}=\frac{1}{1-X^{-\alpha_{i}}}\left(1-s_{i}+s_{i}-X^{-\alpha_{i}} s_{i}\right)=\tilde{\Delta}_{i} .
$$

(b) Using Proposition 3.2e,

$$
\begin{aligned}
q^{-1} T_{s_{i}} X^{\lambda} \mathbf{1}_{0} & =\left(q^{-1} X^{s_{i} \lambda} T_{s_{i}}+\left(1-q^{-2}\right) \frac{X^{\lambda}-X^{s_{i} \lambda}}{1-X^{-\alpha_{i}}}\right) \mathbf{1}_{0} \\
& =\left(X^{s_{i} \lambda}+\left(1-q^{-2}\right) \frac{X^{\lambda}-X^{s_{i} \lambda}}{1-X^{-\alpha_{i}}}\right) \mathbf{1}_{0} \\
& =\left(\frac{X^{s_{i} \lambda}-X^{s_{i} \lambda-\alpha_{i}}+X^{\lambda}-X^{s_{i} \lambda}-q^{-2}\left(X^{\lambda}-X^{s_{i} \lambda}\right)}{1-X^{-\alpha_{i}}}\right) \mathbf{1}_{0} \\
& =\left(\tilde{\Delta}_{i}-q^{-2} \Delta_{i}\right)\left(X^{\lambda}\right) \mathbf{1}_{0},
\end{aligned}
$$


and the first formula in (b) now follows from the second formula in (a). Then

$$
\begin{aligned}
C_{i} & =\left(q^{-2}+\tilde{\Delta}_{i}-q^{-2} \Delta_{i}\right) \\
& =\frac{1}{1-X^{-\alpha_{i}}}\left(q^{-2}-q^{-2} X^{-\alpha_{i}}+1-X^{-\alpha_{i}} s_{i}-q^{-2}+q^{-2} s_{i}\right) \\
& =\frac{1-q^{-2} X^{-\alpha_{i}}}{1-X^{-\alpha_{i}}}+s_{i} \frac{q^{-2}-X^{\alpha_{i}}}{1-X^{\alpha_{i}}}=\left(1+s_{i}\right)\left(\frac{1-q^{-2} X^{-\alpha_{i}}}{1-X^{-\alpha_{i}}}\right) .
\end{aligned}
$$

Remark 4.6. Slightly renormalizing the generators of the affine Hecke algebra by setting $\tilde{T}_{i}=q^{-1} T_{s_{i}}$ allows one to let $q^{-1}=0$ so that $\tilde{T}_{i}$ acts on $\mathbb{K}[P]$ by $\tilde{\Delta}_{i}$. This is the action of the nil affine Hecke algebra on $\mathbb{K}[P]$. Since the $\tilde{T}_{i}$ satisfy the braid relations so do the $\tilde{\Delta}_{i}$. The first formula in Proposition 4.1 shows that $\Delta_{i}$ is a conjugate of $\tilde{\Delta}_{i}$ and so the $\Delta_{i}$ also satisfy the braid relations. Although $C_{i}$ equals $\tilde{\Delta}_{i}$ at $q^{-2}=0$, the operators $C_{i}$ do not satisfy the braid relations. Furthermore, if $w_{0}=s_{i_{1}} \cdots s_{i_{\ell}}$ is a reduced word for the longest element then

$$
C_{i_{1}} \cdots C_{i_{\ell}}=W_{0}\left(q^{-2}\right) \mathbf{1}_{0}+q^{-2}(\text { extra terms }) .
$$

In contrast to the case for Weyl characters (when $q^{-2}=0$ ), because of the $q^{-2}$ (extra terms) the Hall-Littlewood polynomial cannot be generated by applying the product $C_{i_{1}} \cdots C_{i_{\ell}}$ unless one somehow knows how to throw away the extra terms.

Remark 4.7. As operators on $\mathbb{K}[P]$,

$$
\begin{gathered}
q-T_{s_{i}}=\left(\frac{q^{-1}-q X^{-\alpha_{i}}}{1-X^{-\alpha_{i}}}\right)\left(1-s_{i}\right) \quad \text { and } \\
\mathbf{1}_{0}=\frac{1}{W_{0}\left(q^{-2}\right)} \sum_{w \in W} w\left(\prod_{\alpha \in R^{+}} \frac{1-q^{-2} X^{-\alpha}}{1-X^{-\alpha}}\right) .
\end{gathered}
$$

The second formula is equivalent to Macdonald's spherical function formula (4.13).

4.3. Root operators. The idea of root operators is to give an alcove walk interpretation of the action of the operator $C_{i}$ on $\mathbb{K}[P]$ by considering the projections of the alcove walks onto the line perpendicular to $H_{\alpha_{i}}$. The main point is the identity (4.22) which gives a combinatorial description of the action of $C_{i}$ on $\mathbb{K}[P]$. The appropriate combinatorics is more or less forced by the Leibnitz rule or tensor product rule for the operator $C_{i}$ given in (4.28). 
Let $p$ be a positively folded alcove walk.

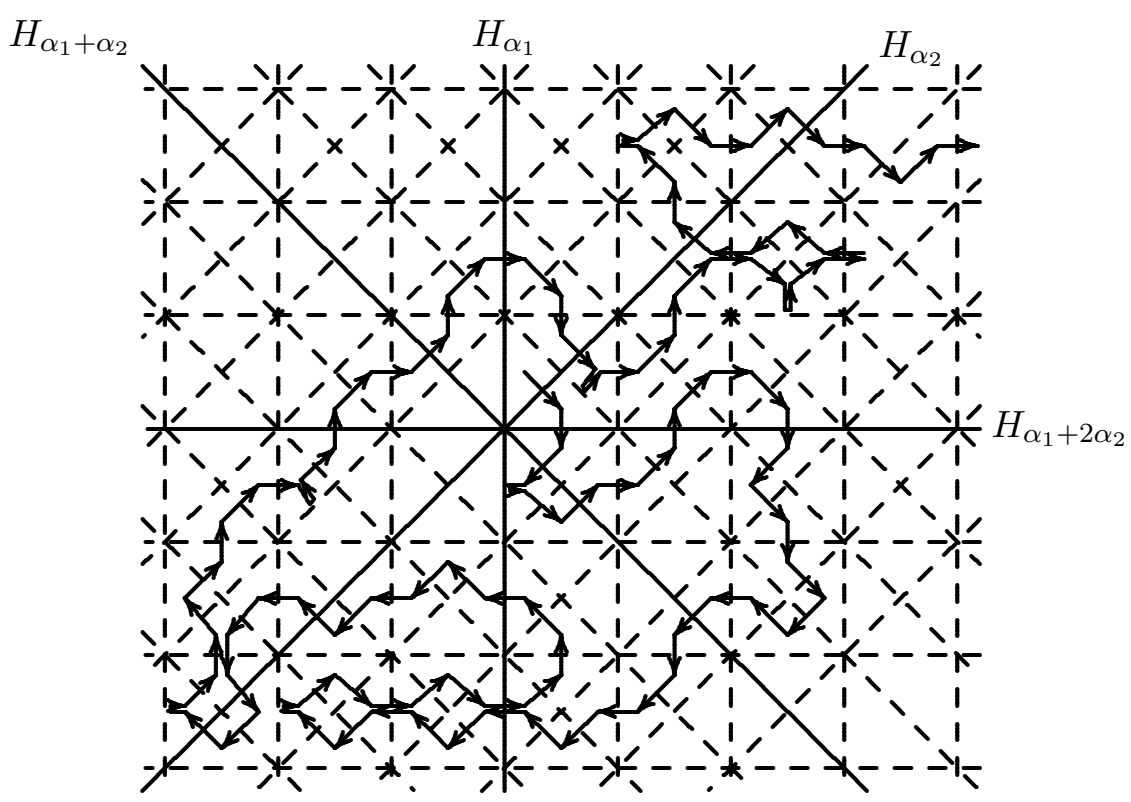

Let $1 \leq i \leq n$. The projection of $p$ onto a line perpendicular to $H_{\alpha_{i}}$ is a positively folded alcove walk $\bar{p}$ "with respect to $\alpha_{i}$ " (the only important information in the projection is the relative position of the walk to each of the hyperplanes parallel to $\left.H_{\alpha_{i}}\right)$.

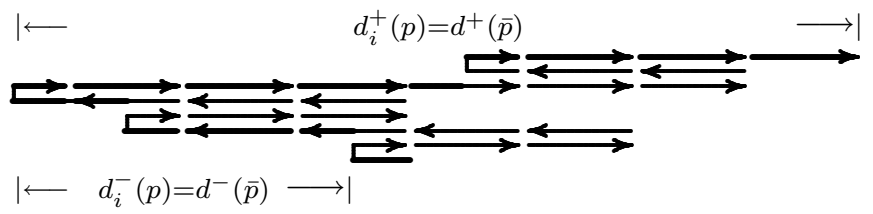

Because $\bar{p}$ is positively folded it is a concatenation of negative-positive sections of the form $c^{-} c^{-} \cdots c^{-} f c^{+} c^{+} \cdots c^{+}$, where $c^{+}$denotes a positive crossing, $c^{-}$a negative crossing, and $f$ a (positive) fold. The outer edge (bottom most negative traveling portion and topmost positive traveling portion) of the walk is a single negative-positive walk

$$
\underbrace{c^{-} c^{-} \cdots c^{-} c^{-}}_{d^{-}(\bar{p}) \text { factors }} f \underbrace{c^{+} c^{+} \cdots c^{+} c^{+}}_{d^{+}(\bar{p}) \text { factors }} .
$$

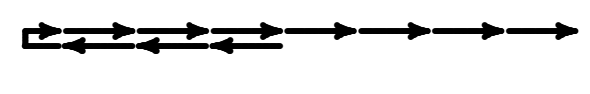

If $\bar{p}$ is the walk in (4.18), the outer edge is the darkened portion of the path, $d^{+}(\bar{p})=7$ and $d^{-}(\bar{p})=3$.

The root operators $\tilde{e}$ and $\tilde{f}$ change the outer edge of the path $\bar{p}$ and leave all other parts of the walk unchanged. Define $\tilde{e} \bar{p}$ and $\tilde{f} \bar{p}$ to be the positively folded 
alcove walks which are the same as $\bar{p}$ except that

$$
\begin{aligned}
& \tilde{f} \bar{p} \text { has outer edge } \underbrace{c^{-} c^{-} \cdots c^{-} c^{-} c^{-}}_{d^{-}(\bar{p})+1 \text { factors }} f \underbrace{c^{+} \cdots c^{+} c^{+}}_{d^{+}(\bar{p})-1 \text { factors }} \text {, and } \\
& \tilde{e} \bar{p} \text { has outer edge } \underbrace{c^{-} c^{-} \cdots c^{-}}_{d^{-}(\bar{p})-1 \text { factors }} f \underbrace{c^{+} c^{+} c^{+} \cdots c^{+} c^{+}}_{d^{+}(\bar{p})+1 \text { factors }}
\end{aligned}
$$

If $\bar{p}$ is the walk in (4.18) then

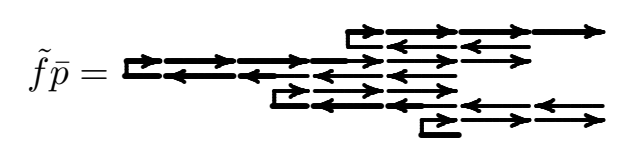

and

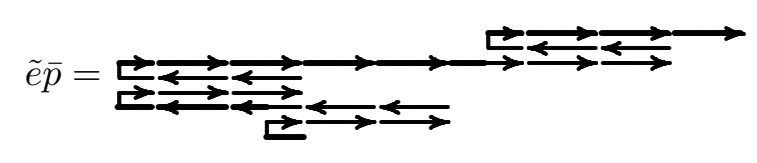

The precise rules for the limiting cases, when $d^{+}(\bar{p})$ or $d^{-}(\bar{p})=0$, are illustrated by the following example, where the dashed arrows indicate the action of $\tilde{e}$ and $\tilde{f}$.

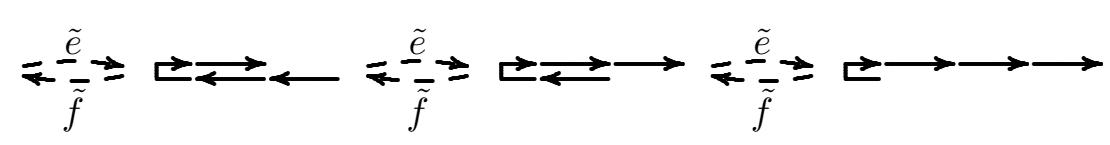

with $\tilde{f}(\longleftarrow \longleftarrow \longleftarrow)=0$ and $\tilde{e}(\longleftarrow \longrightarrow \longrightarrow \longrightarrow)=0$.

With notations for $d^{+}(\bar{p})$ and $d^{-}(\bar{p})$ as in (4.19) and (4.20), define

$$
d_{i}^{+}(p)=d^{+}(\bar{p}) \quad \text { and } \quad d_{i}^{-}(p)=d^{-}(\bar{p})
$$

where $\bar{p}$ is the projection of $p$ onto the line perpendicular to $\alpha_{i}$. The walks $\tilde{e}_{i} p$ and $\tilde{f}_{i} p$ are the walks obtained from $p$ by changing the corresponding edges $p$ (so 
that the projections of $\tilde{e}_{i} p$ and $\tilde{f}_{i} p$ are $\tilde{e} \bar{p}$ and $\tilde{f} \bar{p}$, respectively).
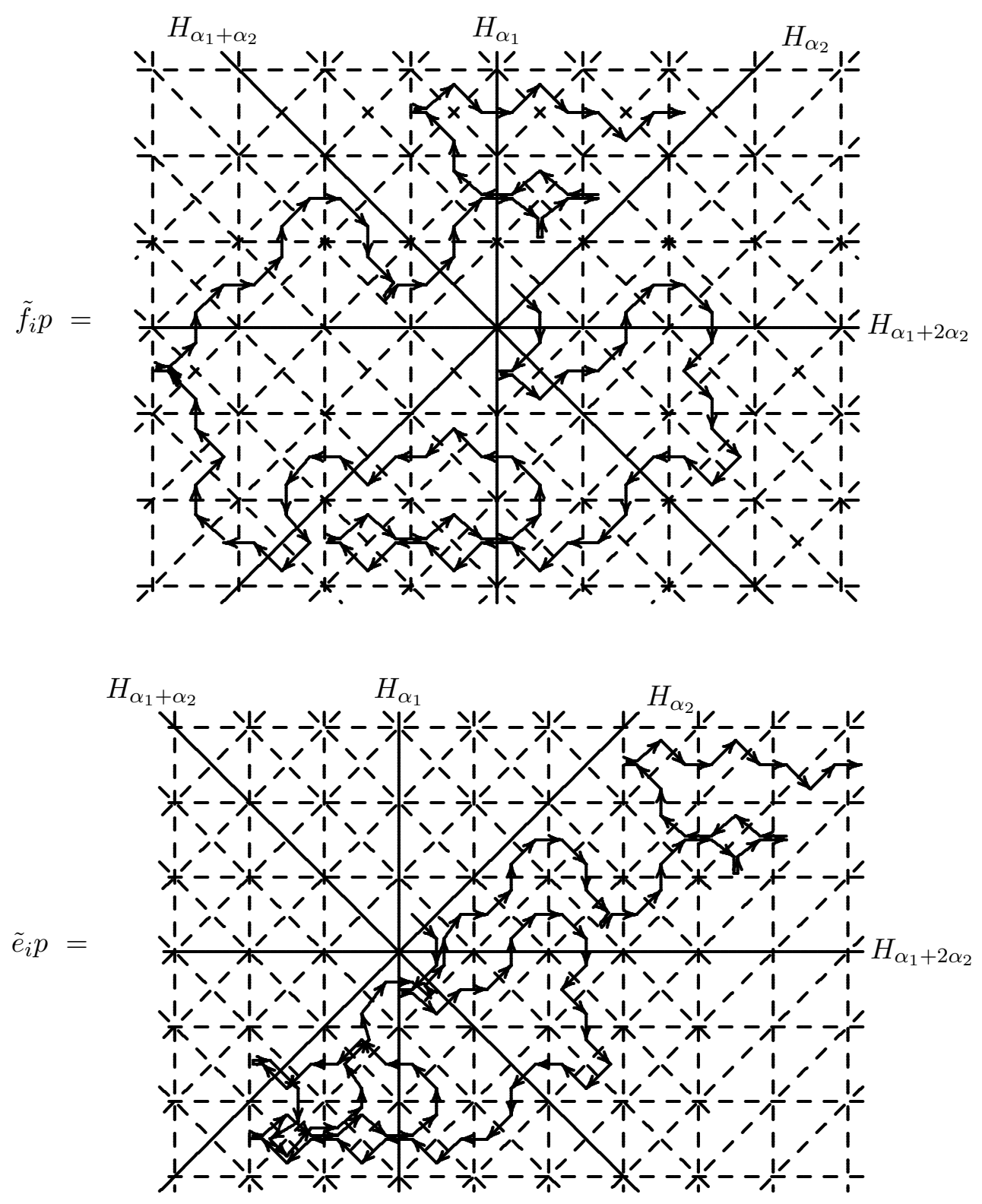

The $i$-string of $p S_{i}(p)$ is the set of paths generated from $p$ by applying the root operators $\tilde{e}_{i}$ and $\tilde{f}_{i}$. The head of the $i$-string is the path $h$ in $S_{i}(p)$ which has $d_{i}^{-}(h)=0$. If $\operatorname{wt}(h)=\lambda$ and $\lambda$ is on the positive side of $H_{\alpha_{i}}$ then

$$
C_{i} X^{\lambda}=q^{-1} X^{s_{i} \lambda} T_{s_{i}}^{-1}+\left(1-q^{-2}\right)\left(X^{s_{i} \lambda}+X^{s_{i} \lambda+\alpha_{i}}+\cdots+X^{\lambda-\alpha_{i}}\right)+X^{\lambda}
$$


and the terms in this sum correspond to the paths in the $i$-string $S_{i}(h)$.

4.4. $q$-Crystals. The $q$-crystals provide a combinatorial model for the spherical Hecke algebra $\mathbf{1}_{0} \tilde{H} \mathbf{1}_{0}$ in the basis of Hall-Littlewood polynomials. Three structural properties motivate the definition of $q$-crystals:

(a) The Hall-Littlewood polynomials are normalized versions of the basis $\mathbf{1}_{0} X^{\lambda} \mathbf{1}_{0}$.

(b) The element $\mathbf{1}_{0}$ is characterized by the property that $C_{i} \mathbf{1}_{0}=\left(1+q^{-2}\right) \mathbf{1}_{0}$ for all $1 \leq i \leq n$.

(c) The action of $C_{i}$ on $\tilde{H} \mathbf{1}_{0} \cong \mathbb{K}[P]$ is captured in the combinatorics of $i$-strings.

These properties indicate that the combinatorics of Hall-Littlewood polynomials can be captured with the root operators.

Let

$$
\begin{gathered}
B_{\text {univ }} \text { be the set of positively folded alcove walks } \\
\text { which begin in the 0-polygon } W A \text {. }
\end{gathered}
$$

If $B$ is a finite subset of $B_{\text {univ }}$ the character of $B$ is

$$
\operatorname{char}(B)=\sum_{p \in B} q^{-(\iota(p)+\varphi(p)-f(p))}\left(1-q^{-2}\right)^{f(p)-c(p)} X^{\mathrm{wt}(p)} .
$$

where $p$ has $f(p)$ folds, $\iota(p) A$ is the alcove where $p$ begins, $\operatorname{wt}(p)+\varphi(p) A$ is the alcove where $p$ ends and

$$
\begin{aligned}
& c(p) \text { the number of folds of } p \\
& \text { touching one of the hyperplanes } H_{\alpha_{1}}, \ldots, H_{\alpha_{n}} \text {. }
\end{aligned}
$$

A $q$-crystal is a finite subset $B$ of $B_{\text {univ }}$ which is closed under the action of the root operators.

A positively folded alcove walk is $i$-dominant if it never touches the hyperplane $H_{\alpha_{i},-1}$. The head $h$ of an $i$-string $S_{i}(p)$ is $i$-dominant and $S_{i}(h)=S_{i}(p)$. A positively folded alcove walk

$$
p \text { is dominant if } p \subseteq C-\rho,
$$

where

$$
C-\rho=\left\{\mu \in \mathfrak{h}_{\mathbb{R}}^{*} \mid\left\langle\mu, \alpha_{i}^{\vee}\right\rangle>-1 \text { for } 1 \leq i \leq n\right\}
$$

In other words, a positively folded alcove walk $p$ is dominant if it is $i$-dominant for all $i, 1 \leq i \leq n$.

Theorem 4.8. Let $B$ be a q-crystal. Then, with notations as in (4.24-4.26),

$$
\operatorname{char}(B)=\sum_{\substack{p \in B \\ p \subseteq C-\rho}} q^{-(\iota(p)+\varphi(p)-f(p))}\left(1-q^{-2}\right)^{f(p)-c(p)} P_{\mathrm{wt}(p)} .
$$


Proof. If $p$ is a dominant walk let

$$
B_{q}(p) \text { be the } q \text {-crystal generated by } p
$$

under the action of the root operators $\tilde{e}_{i}$ and $\tilde{f}_{i}$. The point is that the set of all positively folded alcove walks is partitioned into "equivalence classes" given by the sets $B_{q}(p)$ such that $p \in B_{\text {univ }}$ is dominant and $p$ is the unique dominant walk in $B_{q}(p)$. Because $\mathbf{1}_{0}$ is characterized by the property that $C_{i} \mathbf{1}_{0}=\left(1+q^{-2}\right) \mathbf{1}_{0}$ and the action of $C_{i}$ is modeled by the combinatorics of $i$-strings (4.25), this equivalence relation is generated by the relations $p \sim \tilde{f}_{i} p$ and $p \sim \tilde{e}_{i} p$.

4.5. Products and restrictions. The results in this section are generalizations of the Littlewood-Richardson rules. These are obtained as corollaries of Theorem 4.8 .

The combinatorial definition of the root operators given above is essentially a consequence of the Leibnitz rule for the Demazure operator,

$$
\Delta_{i}(f g)=\Delta_{i}(f) g+\left(s_{i} f\right)\left(\Delta_{i} g\right), \quad \text { for } f, g \in \mathbb{K}[P] .
$$

The corresponding rule for the operators $C_{i}$ is

$$
C_{i}(f g)=\left(C_{i} f\right) g+\left(s_{i} f\right)\left(\left(C_{i}-\left(1+q^{-2}\right)\right) g\right), \quad \text { for } f, g \in \mathbb{K}[P] .
$$

This identity is implicit in the additivity in $\lambda$ of the relation in Proposition 3.2e (the product $T_{s_{i}} X^{\lambda+\mu}=\left(T_{s_{i}} X^{\lambda}\right) X^{\mu}$ can be expanded in two different ways using Proposition 3.2e).

In order to define the product of $p_{1} \otimes p_{2}$ of walks $p_{1}, p_{2} \in B_{\text {univ }}$ the final direction $\varphi\left(p_{1}\right)$ of $p_{1}$ and the initial direction $\iota\left(p_{2}\right)$ of $p_{2}$ need to be taken into account. (To properly model the multiplication of Hall-Littlewood polynomials we must account for the effect of $\mathbf{1}_{0}$ in the product $P_{\mu} \mathbf{1}_{0} P_{\nu} \mathbf{1}_{0}$ and we cannot just concatenate walks as in the alcove walk algebra). Define $p_{1} \otimes p_{2}$, recursively, by

$$
\begin{aligned}
& \text { If } \varphi\left(p_{1}\right)=\iota\left(p_{2}\right) \quad \text { then } \quad p_{1} \otimes p_{2}=p_{1} p_{2}, \text { the concatenation of } p_{1} \text { and } p_{2} \text {, and } \\
& \text { if } \varphi\left(p_{1}\right) \neq \iota\left(p_{2}\right) \quad \text { then } \quad p_{1} \otimes p_{2}=p_{1}^{\prime} \otimes p_{2},
\end{aligned}
$$

where $p_{1}^{\prime}$ is the alcove walk constructed by the following procedure. Let $c_{i_{1}}^{\varepsilon_{1}} \cdots c_{i_{r}}^{\varepsilon_{r}}$ be a minimal length walk from $\varphi\left(p_{1}\right)$ to $\iota\left(p_{2}\right)$. If $\varepsilon_{1}=-$ let $p_{1}^{\prime}=p_{1} c_{i_{1}}^{-}$. If $\varepsilon_{1}=+$ let $H_{\alpha, j}$ be the hyperplane crossed by the last step of $p_{1} c_{i_{1}}^{+}$and change the last negative crossing of $H_{\alpha, j}$ in $p_{1}$ to a fold to obtain a new path $p_{1}^{\prime}$ with $\varphi\left(p_{1}^{\prime}\right)=\varphi\left(p_{1} c_{i_{1}}^{+}\right)$. 
In terms of root operators, the Leibnitz rule (4.28) translates to the property $(4.29)$

$$
\begin{aligned}
& \tilde{e}_{i}\left(p_{1} \otimes p_{2}\right)=\left\{\begin{array}{ll}
\left(\tilde{e}_{i} p_{1}\right) \otimes p_{2}, & \text { if } d_{i}^{+}\left(p_{1}\right) \geq d_{i}^{-}\left(p_{2}\right), \\
p_{1} \otimes\left(\tilde{e}_{i} p_{2}\right), & \text { if } d_{i}^{+}\left(p_{1}\right)<d_{i}^{-}\left(p_{2}\right),
\end{array}\right. \text { and }
\end{aligned}
$$

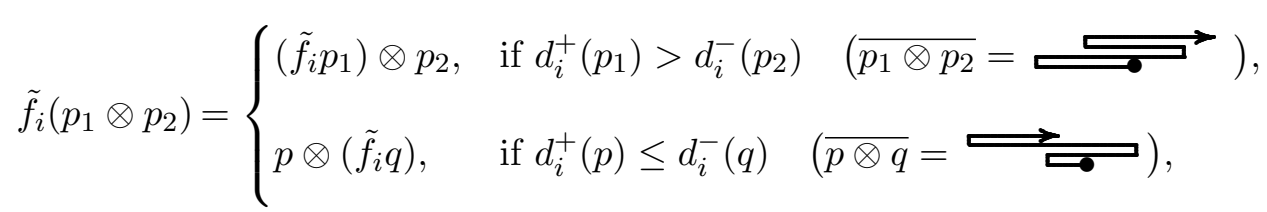

for $p_{1}, p_{2} \in B_{\text {univ }}$. It follows from this version of the Leibnitz rule that if $B_{1}$ and $B_{2}$ are $q$-crystals then the product

$$
B_{1} \otimes B_{2}=\left\{p_{1} \otimes p_{2} \mid p_{1} \in B_{1}, p_{2} \in B_{2}\right\} \quad \text { is also a } q \text {-crystal, }
$$

and

$$
\operatorname{char}\left(B_{1} \otimes B_{2}\right)=\operatorname{char}\left(B_{1}\right) \operatorname{char}\left(B_{2}\right) .
$$

This last property is not completely trivial. The general case follows from the rank one case (projecting onto the line perpendicular to $H_{\alpha}$ ). More importantly, the definition of the product $\otimes$ is forced by (4.29-4.31).

The following theorem is a version of [Sc, Theorem 1.2] and results in [KM] and $[\mathrm{Ha}]$.

Theorem 4.9. Recall the notations from Theorem 4.2. If $\lambda \in P^{+}$let $B_{q}\left(p_{\lambda}\right)$ be the q-crystal generated by $p_{\lambda}$, where $p_{\lambda}$ is a fixed minimal length alcove walk from A to $\lambda+A$. For $\mu, \nu \in P^{+}$,

$$
P_{\mu} P_{\nu}=\sum_{\substack{p \in B_{q}\left(p_{\nu}\right) \\ p \mu \otimes p \subseteq C-\rho}} q^{-(\ell(\iota(p))+\ell(\varphi(p))-f(p))}\left(1-q^{-2}\right)^{f(p)-c(p)} P_{\mu+\mathrm{wt}(p)} .
$$

Proof. Using (4.30) and (4.31) and applying Theorem 4.8 to the $q$-crystal $B_{q}\left(p_{\mu}\right) \otimes$ $B_{q}\left(p_{\nu}\right)$ gives

$$
\begin{aligned}
P_{\mu} P_{\nu} \mathbf{1}_{0} & =\operatorname{char}\left(B_{q}\left(p_{\mu}\right)\right) \operatorname{char}\left(B_{q}\left(p_{\nu}\right)\right) \mathbf{1}_{0}=\operatorname{char}\left(\left(B_{q}\left(p_{\mu}\right) \otimes B_{q}\left(p_{\nu}\right)\right) \mathbf{1}_{0}\right. \\
& =\sum_{\substack{p=p_{1} \otimes p_{2} \in B_{q}\left(p_{\mu}\right) \otimes B_{q}\left(p_{\nu}\right) \\
p_{1} \otimes p_{2} \subseteq C-\rho}} q^{-(\ell(\iota(p))+\ell(\varphi(p))-f(p))}\left(1-q^{-2}\right)^{f(p)-c(p)} P_{\mu+\operatorname{wt}\left(p_{2}\right)} \mathbf{1}_{0}
\end{aligned}
$$

since every path in $p_{1} \in B_{q}\left(p_{\mu}\right)$ which is contained in $C-\rho$ has weight $\mu$ (so that $\left.\mathrm{wt}\left(p_{1} \otimes p_{2}\right)=\mu+\mathrm{wt}\left(p_{2}\right).\right)$

Fix $J \subseteq\{1,2, \ldots, n\}$. The subgroup of $W$ generated by the reflections in the hyperplanes $H_{\alpha_{j}}, j \in J$,

$W_{J}=\left\langle s_{j} \mid j \in J\right\rangle, \quad$ acts on $\mathfrak{h}_{\mathbb{R}}^{*}$, with $C_{J}=\left\{\mu \in \mathfrak{h}_{\mathbb{R}}^{*} \mid\left\langle\mu, \alpha_{j}^{\vee}\right\rangle>0\right.$ for $\left.j \in J\right\}$ 
as a fundamental chamber. Let $H_{J}=\operatorname{span}\left\{T_{w^{-1}}^{-1} \mid w \in W_{J}\right\}$ and let $\mathbf{1}_{J} \in H_{J}$ be given by

$$
\mathbf{1}_{J}^{2}=\mathbf{1}_{J} \quad \text { and } \quad T_{w^{-1}}^{-1} \mathbf{1}_{J}=q^{-\ell(w)} \mathbf{1}_{J}, \quad \text { for } w \in W_{J} .
$$

For $\mu \in P$ let $W_{\mu}^{J}$ be the stabilizer of $\mu$ under the $W_{J}$ action on $P$ and

$$
\begin{aligned}
& \text { define } \quad P_{\mu}^{J}\left(X ; q^{-2}\right) \in \mathbb{K}[P]^{W_{J}} \quad \text { by } \\
& P_{\mu}^{J}\left(X ; q^{-2}\right) \mathbf{1}_{0}=\left(\sum_{w \in W_{J}^{\mu}} q^{-\ell(w)} T_{w^{-1}}^{-1}\right) X^{\mu} \mathbf{1}_{0},
\end{aligned}
$$

where $W_{J}^{\mu}$ is a set of minimal length coset representatives for the cosets in $W_{J} / W_{\mu}^{J}$. Then

$$
\text { up to normalization } P_{\lambda}^{J}\left(X, q^{-2}\right) \mathbf{1}_{0} \text { equals } \mathbf{1}_{J} X^{\lambda} \mathbf{1}_{0},
$$

and

(4.34) $\mathbf{1}_{J} \tilde{H} \mathbf{1}_{0} \quad$ has basis $\quad\left\{P_{\lambda}^{J} \mathbf{1}_{0} \mid \lambda \in P_{J}^{+}\right\}, \quad$ where $P_{J}^{+}=P \cap \overline{C_{J}}$.

with $\overline{C_{J}}=\left\{\mu \in \mathfrak{h}_{\mathbb{R}}^{*} \mid\left\langle\mu, \alpha_{j}^{\vee}\right\rangle>0\right.$ for $\left.j \in J\right\}$.

Theorem 4.10. Recall the notations from Theorem 4.2. If $\mu \in P^{+}$let $B_{q}\left(p_{\mu}\right)$ be the q-crystal generated by $p_{\mu}$, where $p_{\mu}$ is a fixed minimal length alcove walk from $A$ to $\mu+A$. Let $\lambda \in P^{+}$and let $J \subseteq\{1,2, \ldots, n\}$. Then

$$
P_{\lambda}=\sum_{\substack{p \in B(\lambda) \\ p \subseteq C_{J}-\rho_{J}}} q^{-(\ell(\iota(p))+\ell(\varphi(p))-f(p))}\left(1-q^{-2}\right)^{f(p)-c_{J}(p)} P_{\mathrm{wt}(p)}^{J},
$$

where $C_{J}-\rho_{J}=\left\{\mu \in \mathfrak{h}_{\mathbb{R}}^{*} \mid\left\langle\mu, \alpha_{j}^{\vee}\right\rangle>-1\right.$ for $\left.j \in J\right\}$ and $c_{J}(p)$ is the number of folds of $p$ which touch a hyperplane $H_{\alpha_{j}}$ with $j \in J$.

Proof. A $J$-crystal is a set of positively folded alcove walks $B$ which is closed under the operators $\tilde{e}_{j}, \tilde{f}_{j}$, for $j \in J$. Since $P_{\lambda} \mathbf{1}_{0}=\operatorname{char}\left(B_{q}\left(p_{\lambda}\right)\right) \mathbf{1}_{0}$, the statement follows by applying Theorem 4.8 to $B_{q}\left(p_{\lambda}\right)$ viewed as a $J$-crystal.

\section{WEYL CHARACTERS AND CRYSTALS}

Section 5.1 is an exposition of the theory of Weyl characters analogous to the theory of Schur functions in [Mac2, Ch. 1]. The element $a_{\rho}$ in Theorem 5.1 is the Weyl denominator, Lemma 5.2 is a generalization of the Jacobi-Trudi formula and the formulas in Proposition 5.3 are the quantum dimension formula and the Weyl dimension formula, respectively. The results in Proposition 5.4b and 5.4c are the Kostant partition function formula and the Brauer-Klimyk formula, respectively. Sections 5.2-5.6 give an elementary exposition of the theory of crystals and the path model and Section 5.7 explains the relationship between crystals and column strict tableaux. The presentation here is designed to make 
clear the relationship between the general path model and the crystal operators of Lascoux and Schützenberger used in the type A case [LS] (see [Ki] for a nice presentation).

The relationship between the path model used in this section and the alcove walks used in Sections 3 and 4 is as follows. Let $r \in \mathbb{R}_{>0}$. The dilation which replaces the fundamental alcove $A$ by $\frac{1}{r} A$ induces important maps between the corresponding affine Hecke algebras,

$$
\tilde{H}_{A} \hookrightarrow \tilde{H}_{\frac{1}{r} A} \quad \text { and } \quad \tilde{H}_{\frac{1}{r} A} \longrightarrow \tilde{H}_{A},
$$

corresponding to stretching the walks by a factor of $r$. As $r$ gets large the alcove gets small and alcove walks become continuous paths in the limit. At $q^{-1}=0$ the formula for the Hall-Littlewood polynomial in Theorem 4.2a becomes the path model for Weyl characters discovered by P. Littelmann [Li1-3]. In other words, the $q$-crystals become "classical" crystals in the limit.

5.1. Schur functions. Use notations for the Weyl group $W$ and the lattice $P$ as in Section 2. The group algebra of $P$ is the ring

$$
\mathbb{Z}[P] \quad \text { with basis }\left\{X^{\lambda} \mid \lambda \in P\right\} \quad \text { and product } \quad X^{\lambda} X^{\mu}=X^{\lambda+\mu},
$$

for $\lambda, \mu \in P$. The group $W$ acts on $\mathbb{Z}[P]$ by

$$
w X^{\lambda}=X^{w \lambda}, \quad \text { for } w \in W, \lambda \in P .
$$

The ring of symmetric functions and Fock space are

$$
\begin{aligned}
& \mathbb{Z}[P]^{W}=\{f \in \mathbb{Z}[P] \mid w f=f \text { for all } w \in W\} \quad \text { and } \\
& \mathbb{Z}[P]^{\operatorname{det}}=\{f \in \mathbb{Z}[P] \mid w f=\operatorname{det}(w) f \text { for all } w \in W\},
\end{aligned}
$$

respectively. For $\lambda \in P$ define

$$
m_{\lambda}=\sum_{\gamma \in W \lambda} X^{\gamma} \quad \text { and } \quad a_{\lambda}=\sum_{w \in W} \operatorname{det}(w) X^{w \lambda} .
$$

The straightening laws for these elements are

$$
m_{w \lambda}=m_{\lambda} \quad \text { and } \quad a_{w \lambda}=\operatorname{det}(w) a_{\lambda}, \quad \text { for } w \in W \text { and } \lambda \in P .
$$

The second relation implies that $a_{\lambda}=0$ if there exists $w \in W_{\lambda}$ with $\operatorname{det}(w) \neq 1$, and it follows from the straightening laws that

$$
\begin{array}{llll}
\mathbb{Z}[P]^{W} & \text { has basis } & \left\{m_{\lambda} \mid \lambda \in P^{+}\right\}, & \text {and } \\
\mathbb{Z}[P]^{\text {det }} & \text { has basis } & \left\{a_{\lambda+\rho} \mid \lambda \in P^{+}\right\}, &
\end{array}
$$

where $P^{+}$and $\rho$ are as in (2.14) and (2.16), respectively.

The Weyl characters or Schur functions are defined by

$$
s_{\lambda}=\frac{a_{\lambda+\rho}}{a_{\rho}}, \quad \text { for } \lambda \in P .
$$


The following theorem shows that the $s_{\lambda}$ are elements of $\mathbb{Z}[P]$ and that

$$
\mathbb{Z}[P]^{W} \quad \text { has basis } \quad\left\{s_{\lambda} \mid \lambda \in P^{+}\right\} .
$$

Theorem 5.1. Fock space $\mathbb{Z}[P]^{\operatorname{det}}$ is a free $\mathbb{Z}[P]^{W}$ module with generator

$$
a_{\rho}=x^{\rho} \prod_{\alpha \in R^{+}}\left(1-x^{-\alpha}\right) \quad \text { and the map } \begin{aligned}
\mathbb{Z}[P]^{W} & \longrightarrow \mathbb{Z}[P]^{\text {det }} \\
f & \longmapsto a_{\rho} f \\
s_{\lambda} & \longmapsto a_{\lambda+\rho}
\end{aligned}
$$

is a $\mathbb{Z}[P]^{W}$ module isomorphism.

Proof. Let $f \in \mathbb{Z}[P]^{\text {det }}$ and let $\alpha \in R^{+}$. If $f_{\gamma}$ is the coefficient of $x^{\gamma}$ in $f$ then $\sum_{\gamma \in P} f_{\gamma} x^{\gamma}=f=-s_{\alpha} f=\sum_{\gamma \in P}-f_{\gamma} x^{s_{\alpha} \gamma}, \quad$ and so $\quad f=\sum_{\substack{\gamma \in P \\\left\langle\gamma, \alpha^{\vee}\right\rangle \geq 0}} f_{\gamma}\left(x^{\gamma}-x^{s_{\alpha} \gamma}\right)$, since $f_{s_{\alpha} \gamma}=-f_{\gamma}$. Since each term $x^{\gamma}-x^{s_{\alpha} \gamma}$ is divisible $1-x^{-\alpha}, f$ is divisible by $1-x^{-\alpha}$, and thus

$$
\text { each } f \in \mathbb{Z}[P]^{\text {det }} \quad \text { is divisible by } x^{\rho} \prod_{\alpha \in R^{+}}\left(1-x^{-\alpha}\right)
$$

since the polynomials $1-x^{-\alpha}, \alpha \in R^{+}$are coprime in $\mathbb{Z}[P]$ and $x^{\rho}$ is a unit in $\mathbb{Z}[P]$. Comparing coefficients of the maximal terms in $a_{\rho}$ and $x^{\rho} \prod_{\alpha \in R^{+}}\left(1-x^{-\alpha}\right)$ shows that

$$
a_{\rho}=x^{\rho} \prod_{\alpha \in R^{+}}\left(1-x^{-\alpha}\right) .
$$

Thus each $f \in \mathbb{Z}[P]^{\text {det }}$ is divisible by $a_{\rho}$ and so the inverse of multiplication by $a_{\rho}$ is well defined.

The dot action of $S_{n}$ on $P$ is given by

$$
w \circ \mu=w(\mu+\rho)-\rho, \quad \text { for } w \in S_{n}, \mu \in P .
$$

The straightening law

$$
s_{w \circ \mu}=\operatorname{det}(w) s_{\mu}, \quad \text { for } \mu \in P, w \in W .
$$

for the Schur functions follows from the straightening law for the $a_{\mu}$ in (5.3).

Lemma 5.2. Let $f \in \mathbb{Z}[P]^{W}$ and write $f=\sum_{\gamma} f_{\gamma} x^{\gamma}$ so that $f_{\gamma}$ is the coefficient of $x^{\gamma}$ in $f$. Then

$$
f=\sum_{\mu \in P^{+}} f_{\mu} m_{\mu}=\sum_{\lambda \in P^{+}} \eta^{\lambda} s_{\lambda}, \quad \text { where } \quad \eta^{\lambda}=\sum_{w \in W} \operatorname{det}(w) f_{\lambda+\rho-w \rho} .
$$


Proof. The first equality is immediate from the definition of $m_{\mu}$. Since $f \in \mathbb{Z}[P]^{W}$ and the $s_{\lambda}, \lambda \in P^{+}$, are a basis of $\mathbb{Z}[P]^{W}$, the element $f$ can be written as a linear combination of $s_{\lambda}$. Then, since $e^{\lambda+\rho}$ is the unique dominant term in $a_{\lambda+\rho}$,

$$
\begin{aligned}
\eta_{\lambda} & =\left(\text { coefficient of } s_{\lambda} \text { in } f\right)=\left(\text { coefficient of } a_{\lambda+\rho} \text { in } f a_{\rho}\right) \\
& =\left(\text { coefficient of } e^{\lambda+\rho} \text { in } \sum_{\mu \in P} \sum_{w \in W} \operatorname{det}(w) f_{\mu} e^{\mu+w \rho}\right) .
\end{aligned}
$$

Proposition 5.3. If $\nu \in \mathfrak{h}_{\mathbb{R}}^{*}$ and $f=\sum_{\mu \in P} f_{\mu} e^{\mu} \in \mathbb{Z}[P]$ define $f\left(e^{\nu}\right)=\sum_{\mu \in P} f_{\mu} e^{\langle\mu, \nu\rangle}$. Let $\lambda \in P^{+}, t \in \mathbb{R}_{>0}, q=e^{t}$ and $\rho^{\vee}=\frac{1}{2} \sum_{\alpha \in R^{+}} \alpha^{\vee}$. Then

$$
s_{\lambda}\left(q^{\rho^{\vee}}\right)=\prod_{\alpha \in R^{+}} \frac{\left[\left\langle\lambda+\rho, \alpha^{\vee}\right\rangle\right]}{\left[\left\langle\rho, \alpha^{\vee}\right\rangle\right]} \quad \text { and } \quad s_{\lambda}(1)=\prod_{\alpha \in R^{+}} \frac{\left\langle\lambda+\rho, \alpha^{\vee}\right\rangle}{\left\langle\rho, \alpha^{\vee}\right\rangle}
$$

where $[k]=\left(q^{k}-1\right) /(q-1)$ for an integer $k \neq 0$.

Proof.

$$
\begin{aligned}
a_{\lambda+\rho}\left(e^{t \rho^{\vee}}\right) & =\sum_{w \in W} \operatorname{det}(w) e^{\left\langle w(\lambda+\rho), t \rho^{\vee}\right\rangle}=\sum_{w \in W} \operatorname{det}(w) e^{\left\langle w \rho^{\vee}, t(\lambda+\rho)\right\rangle} \\
& =a_{\rho}\left(e^{t(\lambda+\rho)}\right)=e^{\left\langle\rho^{\vee}, t(\lambda+\rho)\right\rangle} \prod_{\alpha \in R^{+}}\left(1-e^{\left\langle-\alpha^{\vee}, t(\lambda+\rho)\right\rangle}\right) .
\end{aligned}
$$

Thus

$$
\begin{aligned}
s_{\lambda}\left(e^{t \rho^{\vee}}\right)=\frac{a_{\lambda+\rho}\left(e^{t \rho^{\vee}}\right)}{a_{\rho}\left(e^{t \rho^{\vee}}\right)} & =\frac{e^{\left\langle\rho^{\vee}, t(\lambda+\rho)\right\rangle}}{e^{\left\langle\rho^{\vee}, t \rho\right\rangle}} \prod_{\alpha \in R^{+}} \frac{1-e^{\left\langle-\alpha^{\vee}, t(\lambda+\rho)\right\rangle}}{1-e^{\left\langle-\alpha^{\vee}, t \rho\right\rangle}} \\
& =q^{-\left\langle\lambda, \rho^{\vee}\right\rangle} \prod_{\alpha \in R^{+}} \frac{q^{\left\langle\lambda+\rho, \alpha^{\vee}\right\rangle}-1}{q^{\left\langle\rho, \alpha^{\vee}\right\rangle}-1}
\end{aligned}
$$

and

$$
s_{\lambda}(1)=\lim _{q \rightarrow 1} s_{\lambda}\left(q^{\rho^{\vee}}\right)=\prod_{\alpha \in R^{+}} \frac{\left\langle\lambda+\rho, \alpha^{\vee}\right\rangle}{\left\langle\rho, \alpha^{\vee}\right\rangle} .
$$

The weight multiplicities are the integers $K_{\lambda \gamma}, \lambda \in P^{+}, \gamma \in P$, defined by the equations

$$
s_{\lambda}=\sum_{\gamma \in P} K_{\lambda \gamma} x^{\gamma}=\sum_{\mu \in P^{+}} K_{\lambda \mu} m_{\mu}
$$


The tensor product multiplicities are the integers $c_{\mu \nu}^{\lambda}, \mu, \nu, \lambda \in P^{+}$, defined by the equations

$$
s_{\mu} s_{\nu}=\sum_{\lambda \in P^{+}} c_{\mu \nu}^{\lambda} s_{\lambda} .
$$

The partition function is the function $p: P \rightarrow \mathbb{Z}_{\geq 0}$ defined by the equation

$$
\prod_{\alpha \in R^{+}} \frac{1}{1-x^{-\alpha}}=\sum_{\gamma \in P} p(\gamma) x^{-\gamma}
$$

Proposition 5.4. Let $\lambda, \mu, \nu \in P^{+}$.

(a) $K_{\lambda \lambda}=1, \quad K_{\lambda, w \mu}=K_{\lambda \mu}$, for $w \in W$, and $\quad K_{\lambda \mu}=0$ unless $\mu \leq \lambda$.

(b) $K_{\lambda \mu}=\sum_{w \in W} \operatorname{det}(w) p(w(\lambda+\rho)-(\mu+\rho))$.

(c) $c_{\mu \nu}^{\lambda}=\sum_{v, w \in W} \operatorname{det}(v w) p(v(\mu+\rho)+w(\nu+\rho)-(\lambda+\rho)-\rho)$.

Proof. (a) The equality $K_{\lambda, w \mu}=K_{\lambda \mu}$ follows from the definition and the fact that $s_{\lambda} \in \mathbb{Z}[P]^{W}$. If $w \neq 1$ then $w(\lambda+\rho)<\lambda+\rho$ so that $w(\lambda+\rho)-\rho<\lambda$ and

$$
\begin{aligned}
s_{\lambda} & =\left(\sum_{w \in W} \operatorname{det}(w) x^{w(\lambda+\rho)-\rho}\right) \cdot \prod_{\alpha \in R^{+}} \frac{1}{1-x^{-\alpha}} \\
& \left.=x^{\lambda}+\text { (lower terms in dominance order }\right) .
\end{aligned}
$$

Thus $K_{\lambda \lambda}=1$ and $K_{\lambda \mu}=0$ unless $\mu \leq \lambda$.

(b) The coefficient of $x^{\mu}$ in

$$
s_{\lambda}=\left(\sum_{w \in W} \operatorname{det}(w) x^{w(\lambda+\rho)-\rho}\right) \prod_{\alpha \in R^{+}} \frac{1}{1-x^{-\alpha}}=\sum_{\substack{w \in W \\ \gamma \in Q^{+}}} \operatorname{det}(w) p(\gamma) x^{w(\lambda+\rho)-\rho-\gamma},
$$

has a contribution $\operatorname{det}(w) p(\gamma)$ when $w(\lambda+\rho)-\rho-\gamma=\mu$ so that $\gamma=w(\lambda+\rho)-$ $(\mu+\rho)$.

(c) Let $\varepsilon=\sum_{w \in W} \operatorname{det}(w) w$. Since $c_{\mu \nu}^{\lambda}$ is the coefficient of $x^{\nu+\rho}$ in

$$
\begin{aligned}
s_{\mu} s_{\nu} a_{\rho} & =\frac{\varepsilon\left(x^{\mu+\rho}\right) \varepsilon\left(x^{\nu+\rho}\right)}{a_{\rho}}=\left(\sum_{v, w \in W} \operatorname{det}(v w) x^{v(\mu+\rho)+w(\nu+\rho)-\rho}\right)\left(\prod_{\alpha \in R^{+}} \frac{1}{1-x^{-\alpha}}\right) \\
& =\sum_{\substack{v, w \in W \\
\gamma \in Q^{+}}} \operatorname{det}(v w) p(\gamma) x^{v(\mu+\rho)+w(\nu+\rho)-\gamma-\rho},
\end{aligned}
$$


there is a contribution $\operatorname{det}(v w) p(\gamma)$ to the coefficient $c_{\mu \nu}^{\lambda}$ when $\lambda+\rho=v(\mu+\rho)+$ $w(\nu+\rho)-\gamma-\rho$ so that $\gamma=v(\mu+\rho)+w(\mu+\rho)-(\lambda+\rho)-\rho$.

Fix $J \subseteq\{1,2, \ldots, n\}$. The subgroup of $W$ generated by the reflections in the hyperplanes $H_{\alpha_{j}}, j \in J$,

$$
\begin{aligned}
& W_{J}=\left\langle s_{j} \mid j \in J\right\rangle, \text { acts on } \mathfrak{h}_{\mathbb{R}}^{*}, \quad \text { with } \\
& C_{J}=\left\{\mu \in \mathfrak{h}_{\mathbb{R}}^{*} \mid\left\langle\mu, \alpha_{j}^{\vee}\right\rangle>0 \text { for } j \in J\right\}
\end{aligned}
$$

as a fundamental chamber. The group $W_{J}$ acts on $P$ and

$$
\mathbb{Z}[P]^{W_{J}}=\left\{f \in \mathbb{Z}[P] \mid w f=f \text { for } w \in W_{J}\right\}
$$

is a subalgebra of $\mathbb{Z}[P]$ which contains $\mathbb{Z}[P]^{W}$. If

$$
\begin{gathered}
\overline{C_{J}}=\left\{\mu \in \mathfrak{h}_{\mathbb{R}}^{*} \mid\left\langle\mu, \alpha_{j}^{\vee}\right\rangle \geq 0 \text { for } j \in J\right\}, \\
P_{J}^{+}=P \cap \overline{C_{J}}, \quad \rho_{J}=\sum_{j \in J} \omega_{j}, \\
a_{\mu}^{J}=\sum_{w \in W_{J}} \operatorname{det}(w) w X^{\mu}, \quad \text { for } \mu \in P, \quad \text { and } \\
s_{\lambda}^{J}=\frac{a_{\lambda+\rho_{J}}^{J}}{a_{\rho_{J}}^{J}}, \quad \text { for } \lambda \in P,
\end{gathered}
$$

then

$$
\left\{s_{\lambda}^{J} \mid \lambda \in P_{J}^{+}\right\} \text {is a basis of } \mathbb{Z}[P]^{W_{J}} .
$$

The restriction multiplicities are the integers $c_{J, \nu}^{\lambda}$ given by

$$
s_{\lambda}=\sum_{\nu \in P_{J}^{+}} c_{J, \nu}^{\lambda} s_{\nu}^{J}
$$

5.2. Paths. Let $\lambda \in P$. The straight line path to $\lambda$ is the map

$$
p_{\lambda}:[0,1] \rightarrow \mathfrak{h}_{\mathbb{R}}^{*} \quad \text { given by } \quad p_{\lambda}(t)=\lambda t .
$$

Let $\ell_{1}, \ell_{2} \in \mathbb{R}_{\geq 0}$. The concatenation of maps $p_{1}:\left[0, \ell_{1}\right] \rightarrow \mathfrak{h}_{\mathbb{R}}^{*}$ and $p_{2}:\left[0, \ell_{2}\right] \rightarrow \mathfrak{h}_{\mathbb{R}}^{*}$ is the map $p_{1} \otimes p_{2}:\left[0, \ell_{1}+\ell_{2}\right] \rightarrow \mathfrak{h}_{\mathbb{R}}^{*}$ given by

$$
\left(p_{1} \otimes p_{2}\right)(t)= \begin{cases}p_{1}(t), & \text { for } t \in\left[0, \ell_{1}\right], \\ p_{1}\left(\ell_{1}\right)+p_{2}\left(t-\ell_{1}\right), & \text { for } t \in\left[\ell_{1}, \ell_{1}+\ell_{2}\right] .\end{cases}
$$

Let $r, \ell \in \mathbb{R}_{\geq 0}$. The $r$-stretch of a map $p:[0, \ell] \rightarrow \mathfrak{h}_{\mathbb{R}}^{*}$ is the map $r p:[0, r \ell] \rightarrow \mathfrak{h}_{\mathbb{R}}^{*}$ given by

$$
(r p)(t)=r \cdot p(t / r)
$$

The reverse of a map $p:[0, \ell] \rightarrow \mathfrak{h}_{\mathbb{R}}^{*}$ is the map $p^{*}:[0, \ell] \rightarrow \mathfrak{h}_{\mathbb{R}}^{*}$ given by

$$
p^{*}(t)=p(\ell-t)-p(\ell) \text {. }
$$


The weight of a map $p:[0, \ell] \rightarrow \mathfrak{h}_{\mathbb{R}}^{*}$ is the endpoint of $p$,

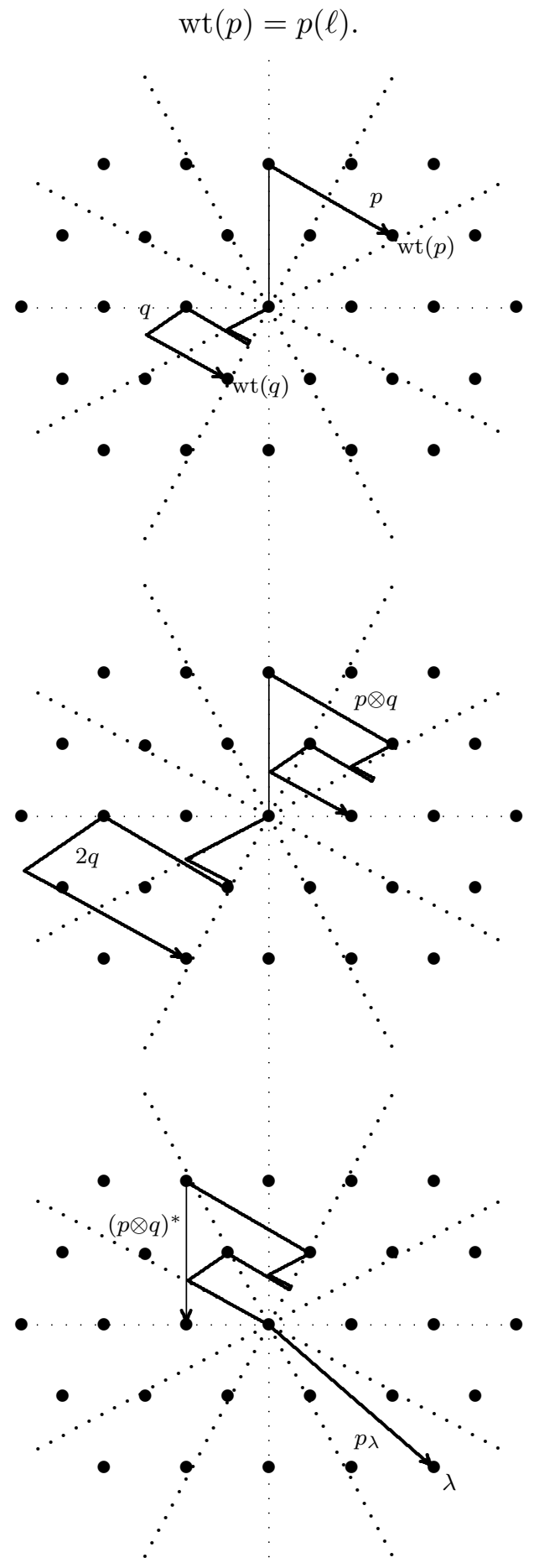


Let

$$
\begin{aligned}
& B_{\text {univ }} \text { be the set of maps generated by the straight line paths } \\
& \text { by operations of concatenation, reversing and stretching. }
\end{aligned}
$$

A path is an element $p:[0, \ell] \rightarrow \mathfrak{h}_{\mathbb{R}}^{*}$ in $B_{\text {univ }}$. Let $B$ be a set of paths (a subset of $\left.B_{\text {univ }}\right)$. The character of $B$ is the element of $\mathbb{Z}[P]$ given by

$$
\operatorname{char}(B)=\sum_{p \in B} X^{\mathrm{wt}(p)}
$$

A crystal is a set of paths $B$ that is closed under the action of the root operators

$$
\begin{aligned}
& \tilde{e}_{i}: B_{\text {univ }} \longrightarrow B_{\text {univ }} \cup\{0\} \\
& \tilde{f}_{i}: B_{\text {univ }} \longrightarrow B_{\text {univ }} \cup\{0\},
\end{aligned}
$$

which are defined and constructed below, in Proposition 5.7 and Theorem 5.8. The crystal graph of $B$ is the graph with

$$
\text { vertices } B \quad \text { and } \quad \text { labeled edges } \quad p^{\prime} \stackrel{i}{\longleftarrow} p \quad \text { if } p^{\prime}=\tilde{f}_{i} p .
$$

5.3. $i$-strings. Let $B$ be a crystal. Let $p \in B$ and fix $i(1 \leq i \leq n)$. The $i$-string of $p$ is the set of paths $S_{i}(p)$ generated from $p$ by applications of the operators $\tilde{e}_{i}$ and $\tilde{f}_{i}$.

The head of $S_{i}(p)$ is $h \in S_{i}(p)$ such that $\tilde{e}_{i} h=0$.

The tail of $S_{i}(p)$ is $t \in S_{i}(p)$ such that $\tilde{f}_{i} t=0$.

The weights of the paths in $S_{i}(p)$ are

$\operatorname{wt}(t)=s_{i} \operatorname{wt}(h)=\operatorname{wt}(h)-\left\langle\operatorname{wt}(h), \alpha_{i}^{\vee}\right\rangle \alpha_{i}, \quad \ldots, \quad \operatorname{wt}(h)-2 \alpha_{i}, \quad \operatorname{wt}(h)-\alpha_{i}, \quad \operatorname{wt}(h)$, and the crystal graph of $S_{i}(p)$ is

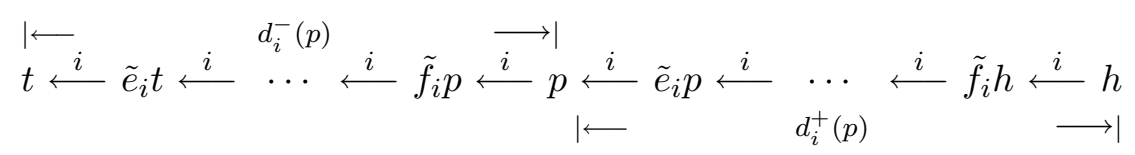

where

$$
d_{i}^{+}(p)=(\text { distance from } h \text { to } p) \quad \text { and } \quad d_{i}^{-}(p)=(\text { distance from } p \text { to } t),
$$

so that $\tilde{e}_{i}^{d_{i}^{+}(p)} p=h$ and $\tilde{f}_{i}^{d_{i}^{-}(p)} p=t$, 
5.4. Highest weight paths. A highest weight path is a path $p$ such that

$$
\tilde{e}_{i} p=0, \quad \text { for all } 1 \leq i \leq n .
$$

A highest weight path is a path $p$ such that, for each $1 \leq i \leq n, p$ is the head of the $i$-string $S_{i}(p)$. Thus $\left\langle p(t), \alpha_{i}^{\vee}\right\rangle>-1$ for all $t$ and all $1 \leq i \leq n$. So a path $p$ is a highest weight path if and only if

$$
p \subseteq C-\rho, \quad \text { where } \quad C-\rho=\{\mu-\rho \mid \mu \in C\} .
$$

Following the example at the end of Section 2, for the root system of type $C_{2}$ the picture is

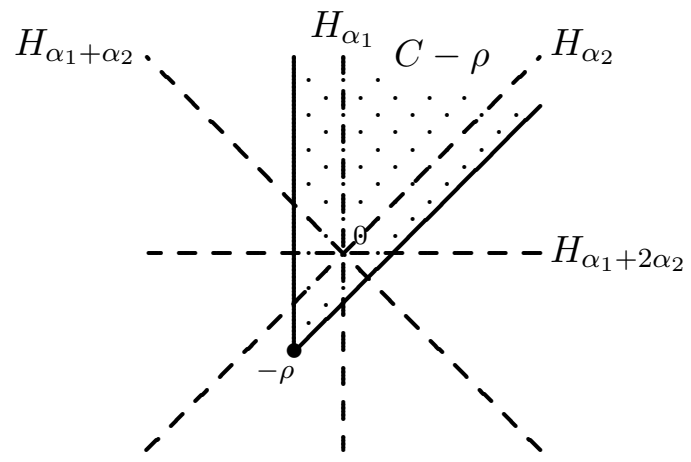

the region $C-\rho$

If $p$ is a highest weight path with $\operatorname{wt}(p) \in P$ then, necessarily, $\operatorname{wt}(p) \in P^{+}$. The following theorem gives an expression for the character of a crystal in terms of the basis $\left\{s_{\lambda} \mid \lambda \in P^{+}\right\}$of $\mathbb{Z}[P]^{W}$.

Theorem 5.5. Let $B$ be a crystal. Let $\operatorname{char}(B)$ be as in (5.24) and $s_{\lambda}$ as in (5.5). Then

$$
\operatorname{char}(B)=\sum_{\substack{p \in B \\ p \subseteq C-\rho}} s_{\mathrm{wt}(p)},
$$

where the sum is over highest weight paths $p \in B$.

Proof. Fix $i, 1 \leq i \leq n$. If $p \in B$ let $s_{i} p$ be the element of the $i$-string of $p$ which satisfies

$$
\operatorname{wt}\left(s_{i} p\right)=s_{i} \operatorname{wt}(p)
$$




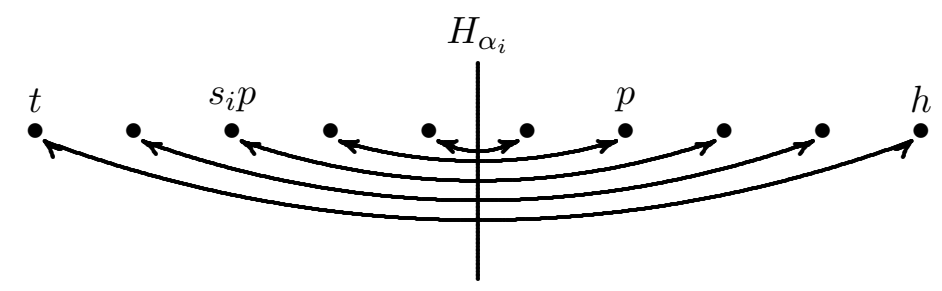

Then $s_{i}\left(s_{i} p\right)=p$ and

$$
s_{i} \operatorname{char}(B)=\sum_{p \in B} X^{s_{i} \mathrm{wt}(p)}=\sum_{p \in B} X^{\mathrm{wt}\left(s_{i} p\right)}=\operatorname{char}(B) .
$$

Hence $\operatorname{char}(B) \in \mathbb{Z}[P]^{W}$.

Let

$$
\varepsilon=\sum_{w \in W} \operatorname{det}(w) w \quad \text { so that } \quad a_{\mu}=\varepsilon\left(X^{\mu}\right), \quad \text { for } \mu \in P .
$$

Since $\operatorname{char}(B) \in \mathbb{Z}[P]^{W}$,

$$
\operatorname{char}(B) a_{\rho}=\operatorname{char}(B) \varepsilon\left(X^{\rho}\right)=\varepsilon\left(\operatorname{char}(B) X^{\rho}\right)
$$

and

$$
\begin{aligned}
\operatorname{char}(B) & =\frac{1}{a_{\rho}} \operatorname{char}(B) a_{\rho}=\frac{\varepsilon\left(\operatorname{char}(B) X^{\rho}\right)}{a_{\rho}} \\
& =\sum_{p \in B} \frac{\varepsilon\left(X^{\mathrm{wt}(p)+\rho}\right)}{a_{\rho}}=\sum_{p \in B} \frac{a_{\mathrm{wt}(p)+\rho}}{a_{\rho}}=\sum_{p \in B} s_{\mathrm{wt}(p)} .
\end{aligned}
$$

There is some cancellation which can occur in this sum. Assume $p \in B$ such that $p \nsubseteq \subseteq C-\rho$ let $t$ be the first time that $p$ leaves the cone $C-\rho$. In other words let $t \in \mathbb{R}_{>0}$ be minimal such that there exists an $i$ with

$$
p(t) \in H_{\alpha_{i},-1} \quad \text { where } \quad H_{\alpha_{i},-1}=\left\{\lambda \in \mathfrak{h}_{\mathbb{R}}^{*} \mid\left\langle\lambda, \alpha_{i}^{\vee}\right\rangle=-1\right\} .
$$

Let $i$ be the minimal index such that the point $p(t) \in H_{\alpha_{i},-1}$ and define $s_{i} \circ p$ to be the element of the $i$-string of $p$ such that

$$
\operatorname{wt}\left(s_{i} \circ p\right)=s_{i} \circ p .
$$




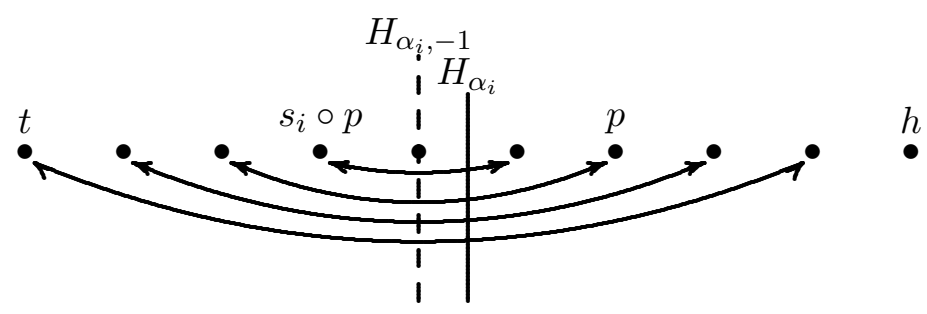

Note that since $\left\langle p_{i}(t), \alpha_{i}^{\vee}\right\rangle=-1, p$ is not the head of its $i$-string and $s_{i} \circ p$ is well defined. If $q=s_{i} \circ p$ then the first time $t$ that $q$ leaves the cone $C-\rho$ is the same as the first time that $p$ leaves the cone $C-\rho$ and $p(t)=q(t)$. Thus $s_{i} \circ q=p$ and $s_{i} \circ\left(s_{i} \circ p\right)=p$. Since

$$
s_{\mathrm{wt}\left(s_{i} \circ p\right)}=s_{s_{i} \circ \mathrm{wt}(p)}=-s_{\mathrm{wt}(p)}
$$

the terms $s_{\mathrm{wt}\left(s_{i} \circ p\right)}$ and $s_{w t(p)}$ cancel in the sum in (5.29). Thus

$$
\operatorname{char}(B)=\sum_{\substack{p \in B \\ p \subseteq C-\rho}} s_{\mathrm{wt}(p)}
$$

Theorem 5.6. Recall the notations for Weyl characters, tensor product multiplicities, restriction multiplicities and paths from (5.5), (5.11), (5.17) and (5.22). For each $\lambda \in P^{+}$fix a highest weight path $p_{\lambda}^{+}$with endpoint $\lambda$ and let

$$
B(\lambda) \text { be the crystal generated by } p_{\lambda}^{+} \text {. }
$$

Let $\lambda, \mu, \nu \in P^{+}$and let $J \subseteq\{1,2, \ldots, n\}$. Then

$$
s_{\lambda}=\sum_{p \in B(\lambda)} X^{\mathrm{wt}(p)}, \quad s_{\mu} s_{\nu}=\sum_{\substack{q \in B(\nu) \\ p_{\mu}^{+} \otimes q \subseteq C-\rho}} s_{\mu+\mathrm{wt}(q)}, \quad \text { and } \quad s_{\lambda}=\sum_{\substack{p \in B(\lambda) \\ p \subseteq C_{J}-\rho_{J}}} s_{\mathrm{wt}(p)}^{J} .
$$

Proof. (a) The path $p_{\lambda}^{+}$is the unique highest weight path in $B(\lambda)$. Thus, by Theorem 5.5, $\operatorname{char}(B(\lambda))=s_{\lambda}$.

(b) By the "Leibnitz formula" for the root operators in Theorem $5.8 \mathrm{c}$ the set

$$
B(\mu) \otimes B(\nu)=\{p \otimes q \mid p \in B(\mu), q \in B(\nu)\}
$$

is a crystal. Since $\operatorname{wt}(p \otimes q)=\operatorname{wt}(p)+\operatorname{wt}(q)$,

$$
\begin{aligned}
s_{\mu} s_{\nu} & =\operatorname{char}(B(\mu)) \operatorname{char}(B(\nu))=\operatorname{char}((B(\mu) \otimes B(\nu)) \\
& =\sum_{\substack{p \otimes q \in B(\mu) \otimes B(\nu) \\
p \otimes q \subseteq C-\rho}} s_{\operatorname{wt}(p)+\operatorname{wt}(q)}=\sum_{\substack{q \in B(\nu) \\
p_{\mu}^{+} \otimes q \subseteq C-\rho}} s_{\mu+\operatorname{wt}(q)},
\end{aligned}
$$


where the third equality is from Theorem 5.5 and the last equality is because the path $p_{\mu}^{+}$has $\operatorname{wt}\left(p_{\mu}^{+}\right)=\mu$ and is the only highest weight path in $B(\mu)$.

(c) A $J$-crystal is a set of paths $B$ which is closed under the operators $\tilde{e}_{j}, \tilde{f}_{j}$, for $j \in J$. Since $s_{\lambda}=\operatorname{char}(B(\lambda))$ the statement follows by applying Theorem 5.5 to $B(\lambda)$ viewed as a $J$-crystal.

\subsection{Root operators for the rank 1 case. Let}

$$
B^{\otimes k}=\left\{b_{1} \otimes \cdots \otimes b_{k} \mid b_{i} \in B\right\}, \quad \text { where } \quad B=\{+1,-1,0\} .
$$

Define

$$
\tilde{f}: B^{\otimes k} \rightarrow B^{\otimes k} \cup\{0\} \quad \text { and } \quad \tilde{e}: B^{\otimes k} \rightarrow B^{\otimes k} \cup\{0\}
$$

as follows. Let $b=b_{1} \otimes \cdots \otimes b_{k} \in B^{\otimes k}$. Ignoring 0s successively pair adjacent unpaired $(+1,-1)$ pairs to obtain a sequence of unpaired -1 s and +1 s

$$
-1-1-1-1-1-1-1+1+1+1+1
$$

(after pairing and ignoring 0s). Then

$$
\begin{aligned}
& \tilde{f} b=\text { same as } b \text { except the leftmost unpaired }+1 \text { is changed to }-1 \text {, } \\
& \tilde{e} b=\text { same as } b \text { except the righttmost unpaired }-1 \text { is changed to }+1 \text {. }
\end{aligned}
$$

If there is no unpaired +1 after pairing then $\tilde{f} b=0$.

If there is no unpaired -1 after pairing then $\tilde{e} b=0$.

These operators coincide with the operators used in the type A case by Lascoux and Schützenberger [LS] (see the nice exposition in [Ki]). The $(+1,-1)$ pairing procedure is equivalent to the process of taking the "outer edge" of the path (4.19-4.20). In the context of Section 4 this is natural since only the outer edge of the path contributes nontrivially to the image of the path in the affine Hecke algebra.

Let $\mathfrak{h}_{\mathbb{R}}^{*}=\mathbb{R}$. By identifying $+1,-1,0$ with the straight line paths

$$
\begin{aligned}
& \stackrel{p_{1}}{\longrightarrow} \stackrel{p_{-1}}{\longleftarrow} \quad p_{0} \\
& p_{1}:[0,1] \rightarrow \mathfrak{h}_{\mathbb{R}}^{*} \quad p_{-1}:[0,1] \rightarrow \mathfrak{h}_{\mathbb{R}}^{*} \quad p_{0}:[0,1] \rightarrow \mathfrak{h}_{\mathbb{R}}^{*} \\
& t \mapsto t, \quad t \mapsto-t, \quad t \mapsto 0,
\end{aligned}
$$

respectively, the set $B^{\otimes k}$ is viewed as a set of maps $p:[0, k] \rightarrow \mathfrak{h}_{\mathbb{R}}^{*}$. Let $B^{\otimes 0}=\{\phi\}$ with $\tilde{f} \phi=0$ and $\tilde{e} \phi=0$. Then

$$
T_{\mathbb{Z}}(B)=\bigsqcup_{k \in \mathbb{Z} \geq 0} B^{\otimes k}
$$


is a set of paths closed under products, reverses and $r$-stretches $\left(r \in \mathbb{Z}_{\geq 0}\right)$ and the action of $\tilde{e}$ and $\tilde{f}$. For $p \in B$ let

$$
\begin{aligned}
& d^{+}(p)=\text { (number of unpaired }+1 \text { s after pairing) } \\
& d^{-}(p)=\text { (number of unpaired }-1 \text { s after pairing). }
\end{aligned}
$$

See the picture in (5.33). These are the nonnegative integers such that $\tilde{f}^{d^{+}(p)} p \neq 0 \quad$ and $\quad \tilde{f}^{d^{+}(p)+1} p=0, \quad$ and $\quad \tilde{e}^{d^{-}(p)} p \neq 0 \quad$ and $\quad \tilde{e}^{d^{-}(p)+1} p=0$.

Proposition 5.7. Use notations for $T_{\mathbb{Z}}(B)$ as in (5.30-5.32).

(a) If $p \in T_{\mathbb{Z}}(B)$ and $\tilde{f} p \neq 0$ then $\tilde{e} \tilde{f} p=p$.

$$
\text { If } p \in T_{\mathbb{Z}}(B) \text { and } \tilde{e} p \neq 0 \text { then } \tilde{f} \tilde{e} p=p \text {. }
$$

(b) If $p \in T_{\mathbb{Z}}(B)$ and $r \in \mathbb{Z}_{\geq 0}$ then

$$
\tilde{f}^{r}(r p)=r(\tilde{f} p) \quad \text { and } \quad \tilde{e}^{r}(r p)=r(\tilde{e} p) .
$$

(c) If $p, q \in T_{\mathbb{Z}}(B)$ then

$$
\tilde{f}(p \otimes q)= \begin{cases}\tilde{f} p \otimes q, & \text { if } d^{+}(p)>d^{-}(q), \\ p \otimes \tilde{f} q, & \text { if } d^{+}(p) \leq d^{-}(q),\end{cases}
$$

and

$$
\tilde{e}(p \otimes q)= \begin{cases}\tilde{e} p \otimes q, & \text { if } d^{+}(p) \geq d^{-}(q), \\ p \otimes \tilde{e} q, & \text { if } d^{+}(p)<d^{-}(q) .\end{cases}
$$

(d) If $p \in T_{\mathbb{Z}}(B)$ then

$$
\tilde{f}\left(p^{*}\right)=(\tilde{e} p)^{*} \quad \text { and } \quad \tilde{e}\left(p^{*}\right)=(\tilde{f} p)^{*} .
$$

Proof. (a), (b) and (d) are direct consequences of the definition of the operators $\tilde{e}$ and $\tilde{f}$ and the definitions of $r$-stretching and reversing.

(c) View $p$ and $q$ as paths. After pairing, $p$ and $q$ have the form

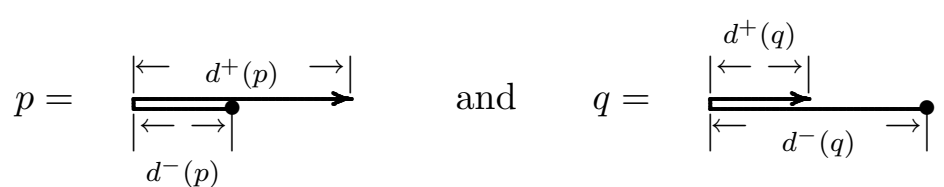

where the left traveling portion of the path corresponds to -1 s and the right traveling portion of the path corresponds to $+1 \mathrm{~s}$. Then

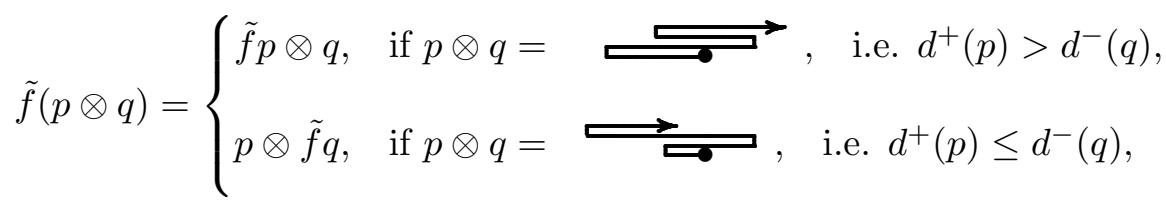


since, in the first case, the leftmost unpaired +1 is from $p$ and, in the second case, it is from $q$.

Use property (b) in Proposition 5.7 to extend the operators $\tilde{e}$ and $\tilde{f}$ to operators on $T_{\mathbb{Q}}(B)$, the set of maps $p:[0, \ell] \rightarrow \mathbb{R}$ generated by $B$ under the operations of concatentation, reversing and $r$-stretching $\left(r \in \mathbb{Q}_{\geq 0}\right)$. Then, by completion, the operators $\tilde{e}$ and $\tilde{f}$ extend to operators on

$T_{\mathbb{R}}(B)$, the set of maps $p:[0, \ell] \rightarrow \mathbb{R}$ generated by $B$ by operations of concatenation, reversing and $r$-stretching $\left(r \in \mathbb{R}_{\geq 0}\right)$.

A rank 1 path is an element of $T_{\mathbb{R}}(B)$.

5.6. The root operators in the general case. Recall from (5.23) that

$B_{\text {univ }}$ is the set of maps generated by the straight line paths by operations of concatenation, reversing and stretching.

and a path is an element $p:[0, \ell] \rightarrow \mathfrak{h}_{\mathbb{R}}^{*}$ in $B_{\text {univ }}$ (see (5.23)).

Let $p:[0, \ell] \rightarrow \mathbb{R}$ be a path and let $\alpha \in R^{+}$be a positive root. The map

$$
p_{\alpha}:[0, \ell] \rightarrow \mathbb{R} \quad \text { given by } \quad p_{\alpha}(t)=\left\langle p(t), \alpha^{\vee}\right\rangle
$$

is a rank 1 path (an element of $T_{\mathbb{R}}(B)$ ). The rank 1 path $p_{\alpha}$ is the projection of $p$ onto the line perpendicular to the hyperplane $H_{\alpha}$. Define operators

$$
\tilde{e}_{\alpha}: B_{\text {univ }} \rightarrow B_{\text {univ }} \cup\{0\} \quad \text { and } \quad \tilde{f}_{\alpha}: B_{\text {univ }} \rightarrow B_{\text {univ }} \cup\{0\}
$$

by

$$
\tilde{e}_{\alpha} p=p+\frac{1}{2}\left(\tilde{e} p_{\alpha}-p_{\alpha}\right) \alpha \quad \text { and } \quad \tilde{f}_{\alpha} p=p-\frac{1}{2}\left(p_{\alpha}-\tilde{f} p_{\alpha}\right) \alpha
$$

and set

$$
\tilde{e}_{i}=\tilde{e}_{\alpha_{i}} \quad \text { and } \quad \tilde{f}_{i}=\tilde{f}_{\alpha_{i}}, \quad \text { for } 1 \leq i \leq n
$$

The operators $\tilde{e}_{i}$ and $\tilde{f}_{i}$ are designed so that after projection onto the line perpendicular to $H_{\alpha_{i}}$ they are the operators $\tilde{e}$ and $\tilde{f}$. 

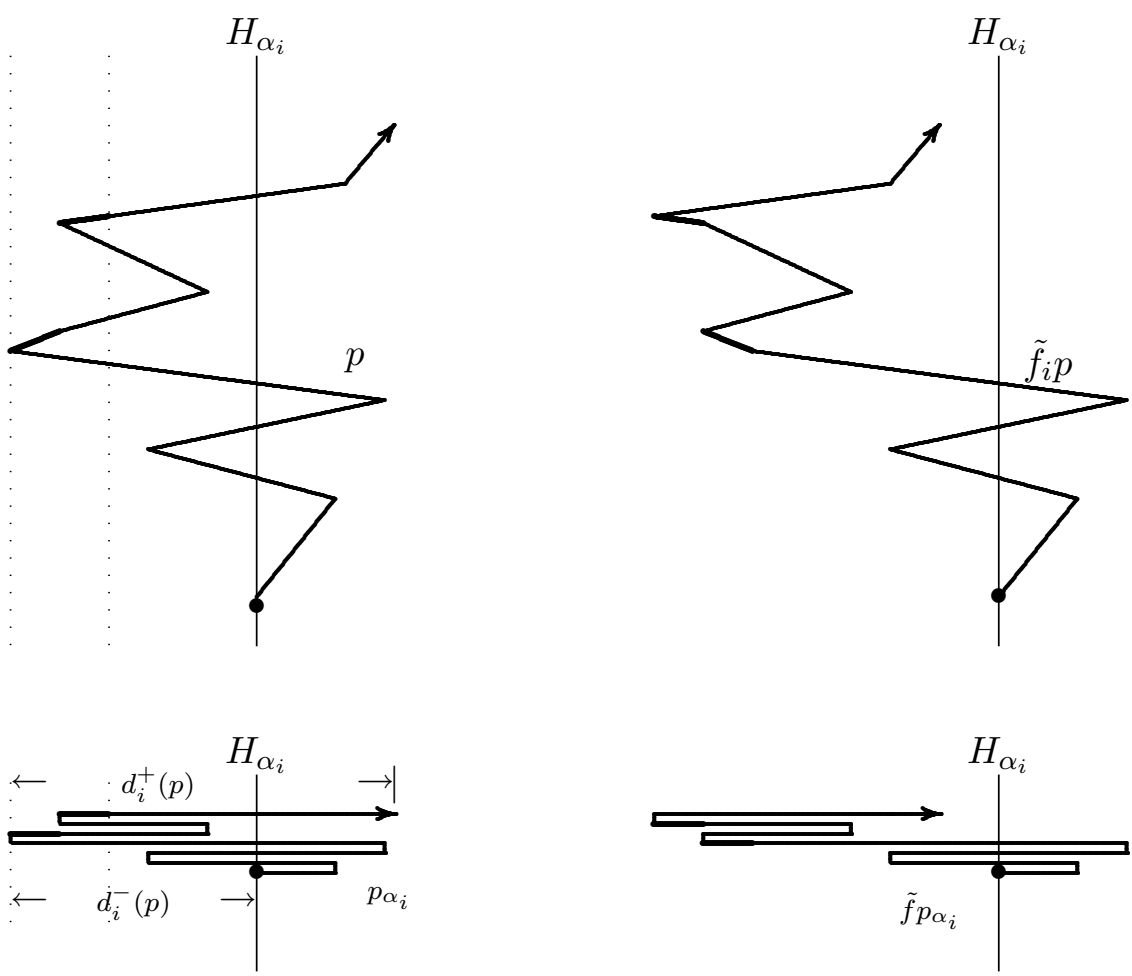

The dark parts of the path $p$ are reflected (in a mirror parallel to $H_{\alpha_{i}}$ ) to form the path $f_{i} p$. The left dotted line is the affine hyperplane parallel to $H_{\alpha_{i}}$ which intersects the path $p$ at its leftmost (most negative) point (relative to $H_{\alpha_{i}}$ ) and the distance between the dotted lines is exactly the distance between lines of lattice points in $P$ parallel to $H_{\alpha_{i}}$.

The following theorem is a consequence of Proposition 5.7 and the definition in (5.34). The uniqueness of the operators $\tilde{f}_{i}$ and $\tilde{e}_{i}$ is forced by the properties (b), (c) and (d) in Theorem 5.8.

Theorem 5.8. The operators $\tilde{e}_{i}$ and $\tilde{f}_{i}$ defined in (5.38) are the unique operators such that

(a) If $p \in B_{\text {univ }}$ and $\tilde{f}_{i} p \neq 0$ then $\tilde{e}_{i} \tilde{f}_{i} p=p$.

If $p \in B_{\text {univ }}$ and $\tilde{e}_{i} p \neq 0$ then $\tilde{f}_{i} \tilde{e}_{i} p=p$.

(b) If $\lambda \in P$ and $\left\langle\lambda, \alpha_{i}^{\vee}\right\rangle \in \mathbb{Z}_{>0}$ then

$$
\tilde{f}_{i}^{\left\langle\lambda, \alpha_{i}^{\vee}\right\rangle} p_{\lambda}=p_{s_{i} \lambda}
$$


(c) If $p, q \in B_{\text {univ }}$ then

$$
\begin{aligned}
& \tilde{f}_{i}(p \otimes q)=\left\{\begin{array}{ll}
\tilde{f}_{i} p \otimes q, & \text { if } d_{i}^{+}(p)>d_{i}^{-}(q), \\
p \otimes \tilde{f}_{i} q, & \text { if } d_{i}^{+}(p) \leq d_{i}^{-}(q),
\end{array} \quad\right. \text { and } \\
& \tilde{e}_{i}(p \otimes q)= \begin{cases}\tilde{e}_{i} p \otimes q, & \text { if } d_{i}^{+}(p) \geq d_{i}^{-}(q), \\
p \otimes \tilde{e}_{i} q, & \text { if } d_{i}^{+}(p)<d_{i}^{-}(q) .\end{cases}
\end{aligned}
$$

where $d_{i}^{ \pm}(p)=d^{ \pm}\left(p_{\alpha_{i}}\right)$ with $d^{ \pm}$as in (5.33) and $p_{\alpha_{i}}$ as in (5.35).

(d) If $p \in B_{\text {univ }}$ and $r \in \mathbb{Z}_{\geq 0}$ then

$$
\tilde{f}_{i}^{r}(r p)=r\left(\tilde{f}_{i} p\right) \quad \text { and } \quad \tilde{e}_{i}^{r}(r p)=r\left(\tilde{e}_{i} p\right) .
$$

(e) If $p \in B_{\text {univ }}$ then

$$
\tilde{f}_{i} p^{*}=\left(\tilde{e}_{i} p\right)^{*} \quad \text { and } \quad \tilde{e}_{i} p^{*}=\left(\tilde{f}_{i} p\right)^{*} .
$$

(f) If $p \in B_{\text {univ }}$ and $\tilde{f}_{i} p \neq 0$ then $\operatorname{wt}\left(\tilde{f}_{i} p\right)=\operatorname{wt}(p)-\alpha_{i}$.

If $p \in B_{\text {univ }}$ and $\tilde{e}_{i} p \neq 0$ then $\operatorname{wt}\left(\tilde{e}_{i} p\right)=\operatorname{wt}(p)+\alpha_{i}$.

5.7. Column strict tableaux. A letter is an element of $B\left(\varepsilon_{1}\right)=\left\{\varepsilon_{1}, \ldots, \varepsilon_{n}\right\}$ and a word of length $k$ is an element of

$$
B\left(\varepsilon_{1}\right)^{\otimes k}=\left\{\varepsilon_{i_{1}} \otimes \cdots \otimes \varepsilon_{i_{k}} \mid 1 \leq i_{1}, \ldots, i_{k} \leq n\right\} .
$$

For $1 \leq i \leq n-1$ define

$$
\tilde{f}_{i}: B\left(\varepsilon_{1}\right)^{\otimes k} \longrightarrow B\left(\varepsilon_{1}\right)^{\otimes k} \cup\{0\} \quad \text { and } \quad \tilde{e}_{i}: B\left(\varepsilon_{1}\right)^{\otimes k} \longrightarrow B\left(\varepsilon_{1}\right)^{\otimes k} \cup\{0\}
$$

as follows. For $b \in B\left(\varepsilon_{1}\right)^{\otimes k}$,

$$
\begin{aligned}
& \text { place }+1 \text { under each } \varepsilon_{i} \text { in } b, \\
& \text { place }-1 \text { under each } \varepsilon_{i+1} \text { in } b, \text { and } \\
& \text { place } 0 \text { under each } \varepsilon_{j}, j \neq i, i+1 .
\end{aligned}
$$

Ignoring 0 s, successively pair adjacent $(+1,-1)$ pairs to obtain a sequence of unpaired -1 s and $+1 \mathrm{~s}$

$$
-1-1-1-1-1-1-1+1+1+1+1
$$

(after pairing and ignoring 0s). Then

$\tilde{f}_{i} b=$ same as $b$ except the letter corresponding to the leftmost unpaired +1 is changed to $\varepsilon_{i+1}$,

$\tilde{e}_{i} b=$ same as $b$ except the letter corresponding to the rightmost unpaired -1 is changed to $\varepsilon_{i}$.

If there is no unpaired +1 after pairing then $\tilde{f}_{i} b=0$. If there is no unpaired -1 after pairing then $\tilde{e}_{i} b=0$. 
A partition is a collection $\lambda$ of boxes in a corner where the convention is that gravity goes up and to the left. As for matrices, the rows and columns of $\lambda$ are indexed from top to bottom and left to right, respectively.

The parts of $\lambda$ are $\lambda_{i}=$ (the number of boxes in row $i$ of $\lambda$ ),

the length of $\lambda$ is $\ell(\lambda)=$ (the number of rows of $\lambda$ ),

the size of $\lambda$ is $\quad|\lambda|=\lambda_{1}+\cdots+\lambda_{\ell(\lambda)}=$ (the number of boxes of $\lambda$ ).

Then $\lambda$ is determined by (and identified with) the sequence $\lambda=\left(\lambda_{1}, \ldots, \lambda_{\ell}\right)$ of positive integers such that $\lambda_{1} \geq \lambda_{2} \geq \cdots \geq \lambda_{\ell}>0$, where $\ell=\ell(\lambda)$. For example,

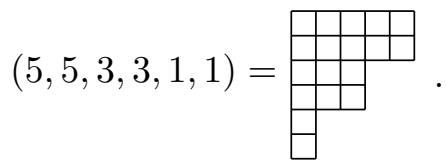

Let $\lambda$ be a partition and let $\mu=\left(\mu_{1}, \ldots, \mu_{n}\right) \in \mathbb{Z}_{\geq 0}^{n}$ be a sequence of nonnegative integers. A column strict tableau of shape $\lambda$ and weight $\mu$ is a filling of the boxes of $\lambda$ with $\mu_{1} 1 \mathrm{~s}, \mu_{2} 2 \mathrm{~s}, \ldots, \mu_{n} n \mathrm{~s}$, such that

(a) the rows are weakly increasing from left to right,

(b) the columns are strictly increasing from top to bottom.

If $p$ is a column strict tableau write $\operatorname{shp}(p)$ and $\operatorname{wt}(p)$ for the shape and the weight of $p$ so that

$$
\begin{aligned}
\operatorname{shp}(p) & =\left(\lambda_{1}, \ldots, \lambda_{n}\right), \quad \text { where } \quad \lambda_{i}=\text { number of boxes in row } i \text { of } p, \quad \text { and } \\
\operatorname{wt}(p) & =\left(\mu_{1}, \ldots, \mu_{n}\right), \quad \text { where } \quad \mu_{i}=\text { number of } i \text { s in } p .
\end{aligned}
$$

For example,

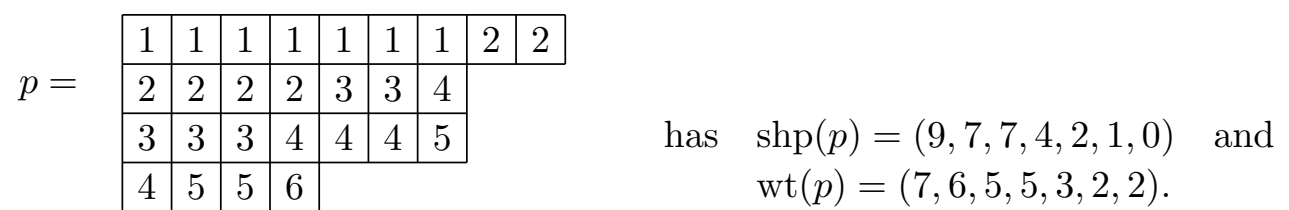

For a partition $\lambda$ and a sequence $\mu=\left(\mu_{1}, \ldots, \mu_{n}\right) \in \mathbb{Z}_{\geq 0}^{n}$ of nonnegative integers write

$$
\begin{aligned}
B(\lambda) & =\{\text { column strict tableaux } p \mid \operatorname{shp}(p)=\lambda\} \\
B(\lambda)_{\mu} & =\{\text { column strict tableaux } p \mid \operatorname{shp}(p)=\lambda \text { and } \operatorname{wt}(p)=\mu\} .
\end{aligned}
$$

Let $\lambda$ be a partition with $k$ boxes and let

$$
B(\lambda)=\{\text { column strict tableaux of shape } \lambda\} .
$$


The set $B(\lambda)$ is a subset of $B\left(\varepsilon_{1}\right)^{\otimes k}$ via the injection

$$
\begin{array}{cl}
B(\lambda) & \hookrightarrow \\
p & \longmapsto(\text { the arabic reading of } p)
\end{array}
$$

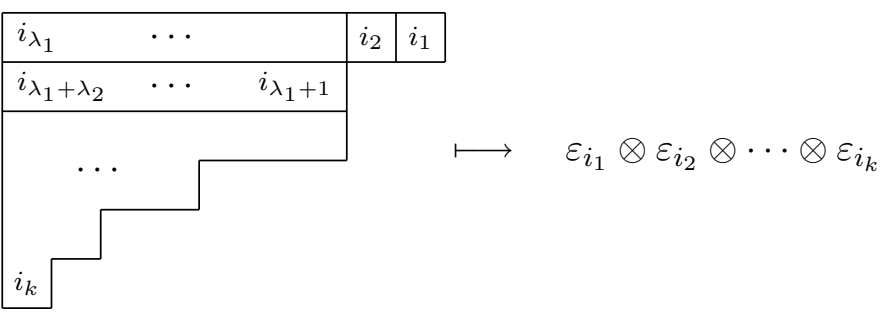

where the arabic reading of $p$ is $\varepsilon_{i_{1}} \otimes \varepsilon_{i_{2}} \otimes \cdots \otimes \varepsilon_{i_{k}}$ if the entries of $p$ are $i_{1}, i_{2}, \ldots, i_{k}$ read right to left by rows with the rows read in sequence beginning with the first row.

Proposition 5.9. Let $\lambda=\left(\lambda_{1}, \ldots, \lambda_{n}\right)$ be a partition with $k$ boxes. Then $B(\lambda)$ is the subset of $B\left(\varepsilon_{1}\right)^{\otimes k}$ generated by

$$
p_{\lambda}=\underbrace{\varepsilon_{1} \otimes \varepsilon_{1} \otimes \cdots \otimes \varepsilon_{1}}_{\lambda_{1} \text { factors }} \otimes \underbrace{\varepsilon_{2} \otimes \varepsilon_{2} \otimes \cdots \otimes \varepsilon_{2}}_{\lambda_{2} \text { factors }} \otimes \cdots \otimes \underbrace{\varepsilon_{n} \otimes \varepsilon_{n} \otimes \cdots \otimes \varepsilon_{n}}_{\lambda_{n} \text { factors }}
$$

under the action of the operators $\tilde{e}_{i}, \tilde{f}_{i}, 1 \leq i \leq n$.

Proof. If $P=P(b)$ is a filling of the shape $\lambda$ then $b_{i_{1}} \otimes \cdots \otimes b_{i_{k}}=b$ is obtained from $P$ by reading the entries of $P$ in arabic reading order (right to left across rows and from top to bottom down the page). The tableau

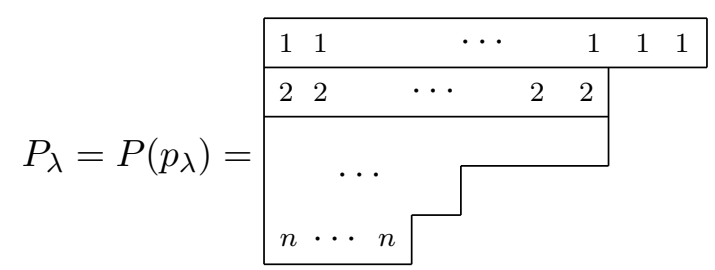

is the column strict tableau of shape $\lambda$ with $1 \mathrm{~s}$ in the first row, $2 \mathrm{~s}$ in the second row, and so on. Define operators $\tilde{e}_{i}$ and $\tilde{f}_{i}$ on a filling of $\lambda$ by

$$
\tilde{e}_{i} P=P\left(\tilde{e}_{i} p\right) \quad \text { and } \quad \tilde{f}_{i} P=P\left(\tilde{f}_{i} b\right), \quad \text { if } P=P(b) .
$$

To prove the proposition we shall show that if $P$ is a column strict tableau of shape $\lambda$ then

(a) $\tilde{e}_{i} P$ and $\tilde{f}_{i} P$ are column strict tableaux,

(b) $P$ can be obtained by applying a sequence of $\tilde{f}_{i}$ to $P_{\lambda}$. 
Let $P^{(j)}$ be the column strict tableau formed by the entries of $P$ which are $\leq j$ and let $\lambda^{(j)}=\operatorname{shp}\left(P^{(j)}\right)$. This conversion identifies $P$ with the sequence

$$
\begin{aligned}
& P=\left(\emptyset=\lambda^{(0)} \subseteq \lambda^{(1)} \subseteq \cdots \subseteq \lambda^{(n)}=\lambda\right), \quad \text { where } \\
& \lambda^{(i)} / \lambda^{(i-1)} \text { is a horizontal strip for each } 1 \leq i \leq n .
\end{aligned}
$$

(a) Let us analyze the action of $\tilde{e}_{i}$ and $\tilde{f}_{i}$ on $P$. The sequence of $+1,-1,0$ constructed via (5.39) is given by

$$
\begin{aligned}
& \text { placing }+1 \text { in each box of } \lambda^{(i)} / \lambda^{(i-1)}, \\
& \text { placing }-1 \text { in each box of } \lambda^{(i+1)} / \lambda^{(i)}, \\
& \text { placing } 0 \text { in each box of } \lambda^{(j)} / \lambda^{(j-1)} \text {, for } j \neq i, i+1,
\end{aligned}
$$

and reading the resulting filling in Arabic reading order. The process of removing $(+1,-1)$ pairs can be executed on the horizontal strips $\lambda^{(i+1)} / \lambda^{(i)}$ and $\lambda^{(i)} / \lambda^{(i-1)}$,

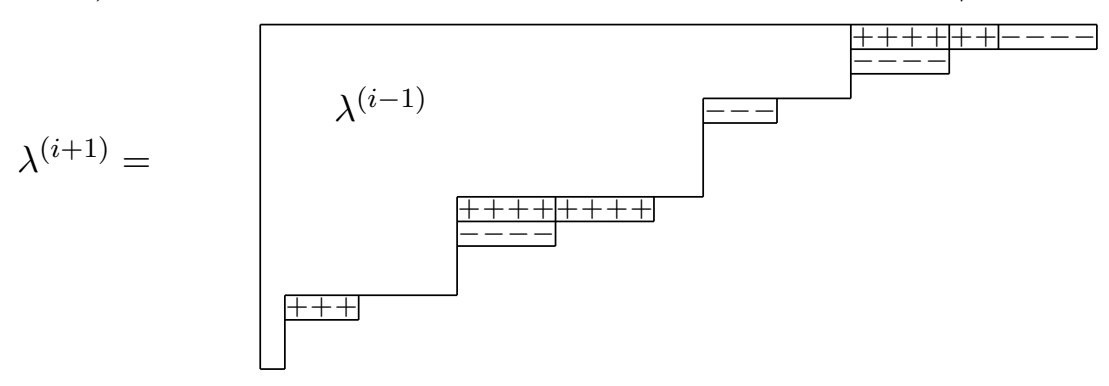

with the effect that the entries in any configuration of boxes of the form

\begin{tabular}{|l|l|l|l|}
\hline+1 & +1 & $\cdots$ & +1 \\
\hline-1 & -1 & $\cdots$ & -1 \\
\hline
\end{tabular}

will be removed. Additional $+1,-1$ pairs will also be removed and the final sequence

$$
\underbrace{-1-1 \cdots-1}_{d_{i}^{-}(p)} \underbrace{+1+1 \cdots+1}_{d_{i}^{+}(p)}
$$

will correspond to a configuration of the form

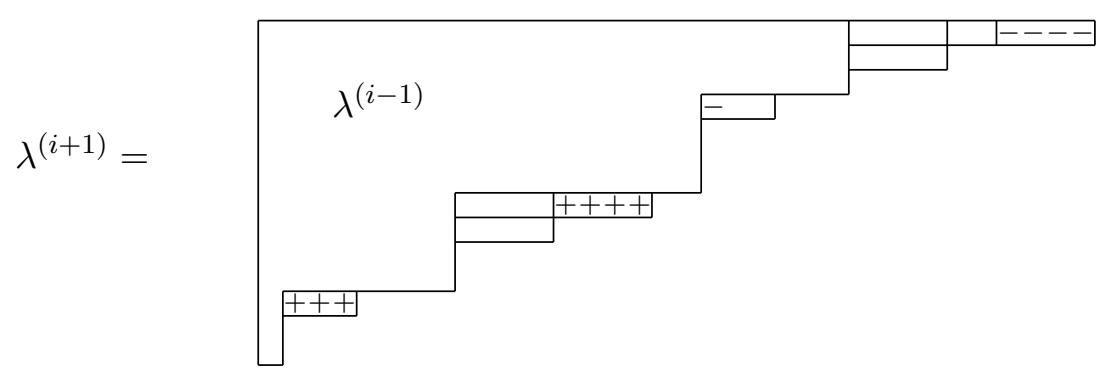

The rightmost -1 in the sequence (5.40) corresponds to a box in $\lambda^{(i+1)} / \lambda^{(i)}$ which is leftmost in its row and which does not cover a box of $\lambda^{(i)} / \lambda^{(i-1)}$. Similarly 
the leftmost +1 in the sequence (5.40) corrresponds to a box in $\lambda^{(i)} / \lambda^{(i-1)}$ which is rightmost in its row and which does not have a box of $\lambda^{(i+1)} / \lambda^{(i)}$ covering it. These conditions guarantee that $\tilde{e}_{i} P$ and $\tilde{f}_{i} P$ are column strict tableaux.

(b) The tableau $P$ is obtained from $P_{\lambda}$ by applying a sequence of $\tilde{f}_{i}$ in the following way. Applying the operator

$$
\tilde{f}_{n, i}=\tilde{f}_{n-1} \cdots \tilde{f}_{i+1} \tilde{f}_{i} \quad \text { to } \quad P_{\lambda}
$$

will change the rightmost $i$ in row $i$ to $n$. A sequence of applications of

$$
\tilde{f}_{n, i}, \quad \text { as } i \text { decreases (weakly) from } n-1 \text { to } 1,
$$

can be used to produce a column strict tableau $P_{n}$ in which

(1) the entries equal to $n$ match the entries equal to $n$ in $P$, and

(2) the subtableau of $P_{n}$ containing the entries $\leq n-1$ is $P_{\lambda^{(n-1)}}$.

Iterating the process and applying a sequence of operators

$$
\tilde{f}_{n-1, i}, \quad \text { as } i \text { decreases (weakly) from } n-2 \text { to } 1,
$$

to the tableau $P_{n}$ can be used to produce a tableau $P_{n-1}$ in which

(1) the entries equal to $n$ and $n-1$ match the entries equal to $n$ and $n-1$ in $P$, and

(2) the subtableau of $P_{n-1}$ containing the entries $\leq n-2$ is $P_{\lambda^{(n-2)}}$.

The tableau $P$ is obtained after a total of $n$ iterations of this process.

\section{REFERENCES}

[BD] Y. Billig and M. Dyer, Decompositions of Bruhat type for the Kac-Moody groups, Nova J. Algebra Geom. 3 no. 1 (1994), 11-31.

[Br] M. Brion, Positivity in the Grothendieck group of complex flag varieties, J. Algebra 258 no. 1 (2002), 137-159.

[GL] S. Gaussent and P. Littelmann, LS galleries, the path model, and MV cycles, Duke Math. J. 127 no. 1 (2005), 35-88.

[GR] S. Griffeth and A. Ram, Affine Hecke algebras and the Schubert calculus, European J. Combin. 25 no. 8 (2004), 1263-1283.

[Ha] T. Haines, Structure constants for Hecke and representation rings, Int. Math. Res. Not. 39 (2003), 2103-2119.

[IM] N. Iwahori and H. Matsumoto, On some Bruhat decomposition and the structure of the Hecke rings of $\mathfrak{p}$-adic Chevalley groups, Inst. Hautes Études Sci. Publ. Math. 25 (1965), $5-48$.

[Ki] K. Killpatrick, A Combinatorial Proof of a Recursion for the q-Kostka Polynomials, J. Comb. Th. Ser. A 92 (2000), 29-53.

[KM] M. Kapovich and J.J. Millson, A path model for geodesics in Euclidean buildings and its applications to representation theory, arXiv: math.RT/0411182.

[LS] A. Lascoux and M.P. Schützenberger, Le monö̈de plaxique, Quad. Ricerce Sci. 109 (1981), $129-156$. 
[LP] C. Lenart and A. Postnikov, Affine Weyl groups in K-theory and representation theory, arXiv: math.RT/0309207.

[Li1] P. Littelmann, A Littlewood-Richardson rule for symmetrizable Kac-Moody algebras, Invent. Math. 116 (1994), 329-346.

[Li2] P. Littelmann, Paths and root operators in representation theory, Ann. Math. 142 (1995), 499-525.

[Li3] P. Littelmann, Characters of representations and paths in $\mathfrak{h}_{\mathbb{R}}^{*}$, Proc. Symp. Pure Math. 61 (1997), 29-49.

[Lu] G. Lusztig, Affine Hecke algebras and their graded version, J. Amer. Math. Soc. 2 (1989), $599-635$

[Mac1] I.G. Macdonald, Spherical functions on a group of p-adic type, Publ. Ramanujan Institute No. 2, Madras (1971).

[Mac2] I.G. Macdonald, Symmetric functions and Hall polynomials, Oxford Mathematical Monographs, Oxford Univ. Press, New York (second edition, 1995)

[PR] H. Pittie and A. Ram, A Pieri-Chevalley formula in the K-theory of a G/B-bundle, Electronic Research Announcements 5 (1999), 102-107, and A Pieri-Chevalley formula for $K(G / B)$, arXiv: math.RT/0401332.

[Sc] C. Schwer, Galleries, Hall-Littlewood polynomials and structure constants of the spherical Hecke algebra, arXiv: math.CO/0506287.

Arun Ram

Department of Mathematics

University of Wisconsin

Madison, WI 53706

E-mail: ram@math.wisc.edu 